\title{
The electromagnetic response in a layered vertical transverse isotropic medium: A new look at an old problem
}

\author{
Jürg Hunziker ${ }^{1}$, Jan Thorbecke ${ }^{1}$, and Evert Slob ${ }^{1}$
}

\begin{abstract}
We determined that the electromagnetic vertical transverse isotropic response in a layered earth can be obtained by solving two equivalent scalar equations, which were for the vertical electric field and for the vertical magnetic field, involving only a scalar global reflection coefficient. Besides the complete derivation of the full electromagnetic response, we also developed the corresponding computer code called EMmod, which models the full electromagnetic fields including internal multiples in the frequency-wavenumber domain and obtains the frequency-space domain solutions through a Hankel transformation by computing the Hankel integral using a 61-point Gauss-Kronrod integration routine. The code is able to model the 3D electromagnetic field in a 1D earth for diffusive methods such as controlled source electromagnetics as well as for wave methods such as ground penetrating radar. The user has complete freedom to place the source and the receivers in any layer. The modeling is illustrated with three examples, which aim to present the different capabilities of EMmod, while assessing its correctness.
\end{abstract}

\section{INTRODUCTION}

Solutions for waves and fields in layered earth models have been published in many forms and over many years. Here, the development of electromagnetic waves and fields is only briefly reviewed. The first to give the procedure for the scalar global reflection coefficient of a layered medium is Airy (1833) who analyzes the formation of Newton's rings in a three-layered medium. For isotropic layered media with a source in the upper half-space, early treatments are given by Wait $(1951,1953)$ and for a horizontal electric dipole over a layered anisotropic half-space by Wait (1966). These results were obtained in the diffusive limit and for isotropic magnetic permeability. Redheffer (1961) introduces a linear fractional transformation, later known as the Redheffer star product, and uses it to formally solve the layered medium problem using the scattering matrix for electromagnetic waves. Kong (1972) solves the layered anisotropic half-space problem for arbitrary dipole sources in the upper half-space and considers both electric and magnetic vertical transverse isotropic (VTI) layers. He exploits the fact that the vertical components of the electric and magnetic fields are independent from each other. He still uses propagation matrices to derive the solutions, by invoking continuity of the horizontal components of the electric and magnetic field vectors. The problem with the propagation matrices method is that in the computational scheme, exponentially growing functions also occur, which makes it unusable for diffusive field models. Redheffer's method uses the scattering matrix, which contains only exponentially damped functions and can be used for both wave and diffusive models. Tsang et al. (1975) solve the VTI-layered-earth problem in the high-frequency limit for microwave passive remote sensing of the earth. A solution for buried sources can be found in Ali and Mahmoud (1979). An excellent review on layered media models is given by Ursin (1983). Kwon and Wang (1986) solve the VTI-layeredearth problem by splitting transverse electric (TE) and transverse magnetic (TM) modes. Xiong (1989) uses vector potentials to solve for the electromagnetic fields in a stratified anisotropic medium. Michalski (2005) derives the Green's tensor in layered VTI media using the transmission-line analogue. The scattering matrix formulation has been used recently to solve the forward problem for arbitrary anisotropic layered media with sources and receivers located in any layer of the model by Loseth and Ursin (2007). Zhong et al. (2008) and Wang et al. (2009) publish algorithms for induction logging tools. A code for forward and inverse modeling of isotropic low frequency electromagnetic data with complete freedom of placement of the source and receiver is published by Key (2009).

Peer-reviewed code related to this article can be found at http://software.seg.org/2015/0001.

Manuscript received by the Editor 8 November 2013; revised manuscript received 21 July 2014; published online 4 December 2014.

${ }^{1}$ Delft University of Technology, Department of Geotechnology, Delft, The Netherlands. E-mail: j.w.hunziker@tudelft.nl; j.w.thorbecke@tudelft.nl; e.c.slob@tudelft.nl.

(C) 2014 Society of Exploration Geophysicists. All rights reserved. 
In this paper, we show that the electromagnetic VTI response can be obtained by solving two equivalent scalar equations involving only a scalar global reflection coefficient. Unlike previous work featuring similar derivations, a corresponding computer code written in $\mathrm{C}$ and Fortran to model electromagnetic fields (EMmod) is attached to this paper. The combination of a simple derivation and a corresponding open-source code that is thoroughly tested (see the "Example" section), opens many possibilities for research and for benchmarking other codes. The code is able to model data for controlled-source electromagnetic (CSEM) methods in the diffusive limit as well as for ground-penetrating radar (GPR) methods for waves in dissipative media. The code allows placement of the source and the receiver anywhere in a stack of VTI layers for any source-receiver component. It outputs the electromagnetic field in the frequency-space domain.

Whenever convenient, the subscript notation is used and the summation convention applies to repeated lowercase subscripts. Latin subscripts take on the values $\{1,2,3\}$, to represent vector directions along the three coordinates in space $\left\{x_{1}, x_{2}, x_{3}\right\}$. Greek subscripts take on the values $\{1,2\}$ to denote vector directions along horizontal coordinates only. The unit matrix is denoted $\delta_{k r}$ defined as $\delta_{k r}=$ 1 if $k=r$ and $\delta_{k r}=0$ otherwise. To denote vector products the Levi-Civita tensor of rank three is used, $\epsilon_{k l m}=-1$ when the sequence $\{k l m\}$ is an odd permutation of $\{123\}, \epsilon_{k l m}=1$ when the sequence $\{k l m\}$ is an even permutation of $\{123\}$, and $\epsilon_{k l m}=$ 0 otherwise. The position vector is denoted $\mathbf{x}=x_{m} \mathbf{i}_{m}=x_{1} \mathbf{i}_{1}+$ $x_{2} \mathbf{i}_{2}+x_{3} \mathbf{i}_{3}$, where $\mathbf{i}_{1}, \mathbf{i}_{2}, \mathbf{i}_{3}$ denote the base vectors of the righthanded Cartesian reference frame. Whenever convenient, the vector $\mathbf{x}$ is written in subscript notation $x_{k}$. Time invariance is exploited by using a time-Laplace transformation defined on a space-time dependent vector function $\mathbf{E}(\mathbf{x}, t)$ as

$$
\hat{\mathbf{E}}(\mathbf{x}, s)=\int_{t=0}^{\infty} \mathbf{E}(\mathbf{x}, t) \exp (-s t) \mathrm{d} t
$$

where $s$ denotes the Laplace transformation variable, which can be taken real and positive, or $s=\mathrm{i} \omega$, with $\omega=2 \pi f, f$ being the natural frequency. Horizontal shift-invariance, as present in a $1 D$ medium, allows carrying out a 2D spatial Fourier transformation, and it is defined on a Laplace-transformed vector function $\hat{\mathbf{E}}(\mathbf{x}, s)$ as

$$
\tilde{\mathbf{E}}\left(\mathbf{k}_{T}, z, s\right)=\int_{\mathbf{x}_{T}=-\infty}^{\infty} \hat{\mathbf{E}}(\mathbf{x}, s) \exp \left(-\mathrm{i} k_{\alpha} x_{\alpha}\right) \mathrm{d}^{2} \mathbf{x}_{T},
$$

where the subscript $T$ is used to indicate the horizontal vector and $k_{\alpha}$ denotes the two components of the horizontal wavenumber vector.

The horizontal and vertical conductivities are denoted as $\eta, \eta^{(v)}$ and $\eta=\sigma+s \varepsilon, \eta^{(v)}=\sigma^{(v)}+s \varepsilon^{(v)}$, where $\sigma, \sigma^{(v)}$ are the horizontal and vertical electric conductivity, $\varepsilon, \varepsilon^{(v)}$ are the horizontal and vertical electric permittivity. The horizontal and vertical transverse resistivity functions are denoted as $\zeta, \zeta^{(v)}$ and $\zeta=s \mu, \zeta^{(v)}=s \mu^{(v)}$, with magnetic permeability $\mu$. The wavenumbers related to the vertical conductivity and to the transverse resistivity, are denoted $\gamma, \bar{\gamma}$ with $\gamma=\sqrt{\zeta \eta^{(v)}}$ and $\bar{\gamma}=\sqrt{\zeta^{(v)} \eta}$.

The configuration has an electric or magnetic current type source located in a layer of finite thickness that is embedded in two different layered half-spaces. The medium is a stack of $N$ layers of finite thickness that is bounded at the top and bottom by homogeneous half-spaces. For example, the upper half-space can act as a model for air, and the lower half-space can be taken to lie outside the zone of interest, such that it can act as a radiation boundary condition. This stack of layers is a model for a 1D layered earth. Each layer is VTI characterized by horizontal electric and magnetic parameters that differ from the vertical ones. This type of anisotropy allows complete splitting between TE and TM modes. The solution is obtained in a form that requires the least amount of derivations and the corresponding code requires the least amount of numerical operations.

In a horizontally layered VTI earth model the vertical direction is the only direction in which the medium parameters are piecewise constant, while in both horizontal directions they are constant. The vertical direction is therefore chosen as the direction of reference to determine TE modes and TM modes. The TE mode has no vertical electric field component and the TM mode has no vertical magnetic field component. The TE mode and TM mode wave field equations are therefore independently obtained for the vertical components of the magnetic and electric field strengths, respectively. Here, all electromagnetic Green's functions are derived in the horizontal wavenumber domain for the electric and magnetic fields generated by an electric or magnetic point source somewhere in the layered VTI medium. The receiver is also located somewhere in the VTI medium. Once all Green's functions are found, the electric field generated by an electric dipole is derived in the space frequency domain by means of an integral equation. Finally, we illustrate the solution method using numerical examples.

\section{PROPERTIES OF THE ELECTROMAGNETIC FIELD AND THE REDUCTION OF WORK}

Maxwell's equations can be written in the space-Laplace transformed domain as

$$
\begin{array}{r}
-\epsilon_{k m p} \partial_{m} \hat{H}_{p}(\mathbf{x}, s)+\eta_{k r}(\mathbf{x}, s) \hat{E}_{r}(\mathbf{x}, s)=-\hat{J}_{k}^{e}(\mathbf{x}, s), \\
\epsilon_{j n r} \partial_{n} \hat{E}_{r}(\mathbf{x}, s)+\zeta_{j p}(\mathbf{x}, s) \hat{H}_{p}(\mathbf{x}, s)=-\hat{J}_{j}^{m}(\mathbf{x}, s),
\end{array}
$$

which are valid for arbitrary anisotropic media with relaxation and losses. If we substitute $\hat{\mathbf{E}}(\mathbf{x}, s)=\hat{\mathbf{H}}(\mathbf{x}, s), \eta_{m n}(\mathbf{x}, s)=-\zeta_{m n}(\mathbf{x}, s)$, and $\hat{\mathbf{J}}^{e}(\mathbf{x}, s)=-\hat{\mathbf{J}}^{m}(\mathbf{x}, s)$, the same Maxwell equations are obtained. This is known as the duality principle. This implies that for any partial or full solution of a boundary value problem that is found for the electric field vector $\hat{\mathbf{E}}(\mathbf{x}, s)$, or a component thereof, the solution for the magnetic field vector $\hat{\mathbf{H}}(\mathbf{x}, s)$ or the same component thereof can be written down using these substitutions. To reduce bookkeeping, source-receiver reciprocity can be used. Let the Green's tensor functions related to an electric current point dipole satisfy the following equations:

$$
\begin{gathered}
-\epsilon_{k m p} \partial_{m} \hat{G}_{p s}^{m e}\left(\mathbf{x}, \mathbf{x}^{\prime}, s\right)+\eta_{k r}(\mathbf{x}, s) \hat{G}_{r s}^{e e}\left(\mathbf{x}, \mathbf{x}^{\prime}, s\right) \\
=-\delta_{k s}\left(\mathbf{x}-\mathbf{x}^{\prime}\right), \\
\epsilon_{j n r} \partial_{n} \hat{G}_{r q}^{e e}\left(\mathbf{x}, \mathbf{x}^{\prime}, s\right)+\zeta_{j p}(\mathbf{x}, s) \hat{G}_{p q}^{m e}\left(\mathbf{x}, \mathbf{x}^{\prime}, s\right)=0, \\
-\epsilon_{k m p} \partial_{m} \hat{G}_{p s}^{m m}\left(\mathbf{x}, \mathbf{x}^{\prime}, s\right)+\eta_{k r}(\mathbf{x}, s) \hat{G}_{r s}^{e m}\left(\mathbf{x}, \mathbf{x}^{\prime}, s\right)=0,
\end{gathered}
$$




$$
\begin{aligned}
& \epsilon_{j n r} \partial_{n} \hat{G}_{r q}^{e m}\left(\mathbf{x}, \mathbf{x}^{\prime}, s\right)+\zeta_{j p}(\mathbf{x}, s) \hat{G}_{p q}^{m m}\left(\mathbf{x}, \mathbf{x}^{\prime}, s\right) \\
& \quad=-\delta_{j q}\left(\mathbf{x}-\mathbf{x}^{\prime}\right)
\end{aligned}
$$

source-receiver reciprocity then states:

$$
\begin{gathered}
\hat{G}_{r s}^{e e}\left(\mathbf{x}, \mathbf{x}^{\prime}, s\right)=\hat{G}_{s r}^{e e}\left(\mathbf{x}^{\prime}, \mathbf{x}, s\right), \\
\hat{G}_{r q}^{e m}\left(\mathbf{x}, \mathbf{x}^{\prime}, s\right)=-\hat{G}_{q r}^{m e}\left(\mathbf{x}^{\prime}, \mathbf{x}, s\right) .
\end{gathered}
$$

In these equations the Green's tensors have superscripts, in which the first superscript denotes the field type and the second superscript denotes the source type. Similarly, the first subscript denotes the field vector component and the second subscript denotes the source vector component. From the duality principle, it was already established that

$$
\begin{aligned}
& \hat{G}_{p q}^{m m}\left(\mathbf{x}, \mathbf{x}^{\prime}, s, \eta_{k r}, \zeta_{i j}\right)=-\hat{G}_{p q}^{e e}\left(\mathbf{x}, \mathbf{x}^{\prime}, s,-\zeta_{k r},-\eta_{i j}\right), \\
& \hat{G}_{p q}^{m e}\left(\mathbf{x}, \mathbf{x}^{\prime}, s, \eta_{k r}, \zeta_{i j}\right)=-\hat{G}_{p q}^{e m}\left(\mathbf{x}, \mathbf{x}^{\prime}, s,-\zeta_{k r},-\eta_{i j}\right) .
\end{aligned}
$$

From these results, it is clear that the solution for the magnetic field Green's functions are known when those for the electric fields have been found. Moreover, knowing six of the nine components of the Green's tensor representing the electric field generated by an electric current dipole suffices to know all components of the Green's tensor describing the electric field generated by an electric source dipole and at the same time all components of the Green's tensor describing the magnetic field generated by a magnetic source dipole.

To break down the problem, first, Maxwell's equations are transformed to the horizontal wavenumber domain. This simplifies the analysis because Maxwell's equations are reduced to one scalar second-order differential equation for the vertical electric field component and one for the vertical magnetic field component. This leads to further reduction in work because the whole electromagnetic field is known when the vertical component of the electric field is known for all sources. This is demonstrated in the next section.

\section{DECOMPOSITION OF THE ELECTROMAGNETIC FIELD AND THE FREQUENCY WAVENUMBER DOMAIN HOMOGENEOUS SPACE GREEN'S FUNCTIONS}

We start with the solution in a homogeneous space, but we use the coupled Maxwell equations in the horizontal wavenumber domain to prepare for the layered medium treated afterward. They are given by

$$
\begin{gathered}
\eta^{(v)} \tilde{E}_{3}=-\tilde{J}_{3}^{e}-\epsilon_{3 \lambda \beta} \mathrm{i} k_{\lambda} \tilde{H}_{\beta}, \\
\zeta^{(v)} \tilde{H}_{3}=-\tilde{J}_{3}^{m}+\epsilon_{3 \lambda \beta} \mathrm{i} k_{\lambda} \tilde{E}_{\beta}, \\
\eta \tilde{E}_{\alpha}=-\tilde{J}_{\alpha}^{e}+\epsilon_{\alpha 3 \beta} \partial_{3} \tilde{H}_{\beta}-\epsilon_{\alpha \lambda 3} \mathrm{i} k_{\lambda} \tilde{H}_{3}, \\
\zeta \tilde{H}_{\beta}=-\tilde{J}_{\beta}^{m}-\epsilon_{\beta 3 \alpha} \partial_{3} \tilde{E}_{\alpha}+\epsilon_{\beta \lambda 3} \mathrm{i} k_{\lambda} \tilde{E}_{3} .
\end{gathered}
$$

The horizontal electric field components are eliminated by substituting the expression of equation 15 into 16 :

$$
\left[\eta \zeta-\partial_{3} \partial_{3}\right] \tilde{H}_{\alpha}=-\eta \tilde{J}_{\alpha}^{m}+\epsilon_{\alpha 3 \beta} \partial_{3} \tilde{J}_{\beta}^{e}+\mathrm{i} k_{\alpha} \partial_{3} \tilde{H}_{3}+\eta \epsilon_{\alpha \lambda 3} \mathrm{i} k_{\lambda} \tilde{E}_{3},
$$

and using equation 17 in equation 13 , to obtain

$$
\begin{gathered}
\left(\eta \zeta-\partial_{3} \partial_{3}\right) \eta^{(v)} \tilde{E}_{3}=\left(\partial_{3} \partial_{3}-\eta \zeta\right) \tilde{J}_{3}^{e}+\eta \epsilon_{3 \alpha \beta} \mathrm{i} k_{\alpha} \tilde{J}_{\beta}^{m} \\
-\epsilon_{3 \alpha \beta} \mathrm{i} k_{\alpha}\left(\epsilon_{\beta 3 \alpha} \partial_{3} \tilde{J}_{\alpha}^{e}+\mathrm{i} k_{\beta} \partial_{3} \tilde{H}_{3}+\eta \epsilon_{\beta \lambda 3} \mathrm{i} k_{\lambda} \tilde{E}_{3}\right)
\end{gathered}
$$

leading to the following equation for the vertical electric field component

$$
\left(\partial_{3} \partial_{3}-\Gamma^{2}\right) \eta^{(v)} \tilde{E}_{3}=\eta \zeta \tilde{J}_{3}^{e}+\partial_{3}\left(\mathrm{i} k_{\alpha} \tilde{J}_{\alpha}^{e}-\partial_{3} \tilde{J}_{3}^{e}\right)-\eta \epsilon_{3 \alpha \beta} \mathrm{i} k_{\alpha} \tilde{J}_{\beta}^{m},
$$

with vertical wavenumber related to vertical conductivity given by $\Gamma=\sqrt{\eta / \eta^{(v)}} \sqrt{\kappa^{2}+\gamma^{2}}$, with $\kappa=\left(k_{1}^{2}+k_{2}^{2}\right)^{1 / 2}$ being the horizontal radial wavenumber, and $\mathfrak{R}\{\Gamma\} \geq 0$. To arrive at equations 17 and 19 , the epsilon-delta identity has been used,

$$
\epsilon_{k m p} \epsilon_{p n r}=\delta_{k n} \delta_{m r}-\delta_{k r} \delta_{m n}
$$

Without loss of generality, we take the sources to be point sources, defined by

$$
\left\{\tilde{J}_{k}^{e}, \tilde{J}_{k}^{m}\right\}=\left\{\hat{I}_{k}^{e}(s), \hat{I}_{k}^{m}(s)\right\} \delta\left(x_{3}-x_{3}^{S}\right),
$$

with the source position vector given by $x_{3}^{S}=\left\{0,0, z^{S}\right\}$ and the frequency spectrum of the source specified by $\hat{I}_{k}^{e, m}(s)$. The two corresponding Green's functions are solutions of modified Helmholtz equations,

$$
\left(\partial_{3} \partial_{3}-\Gamma^{2}\right) \tilde{G}=-\delta\left(x_{3}-x_{3}^{S}\right) .
$$

The solutions to these equations are well known, and given by

$$
\tilde{G}\left(x_{3}-x_{3}^{S}\right)=\frac{\exp (-\Gamma h)}{2 \Gamma},
$$

where $h=\left|x_{3}-x_{3}^{S}\right|$. Equation 23 is the TM-mode Green's function. In view of equations 19,21 , and 23 , the vertical component of the electric field is given by

$$
\begin{aligned}
\eta^{(v)} \tilde{E}_{3}= & \left(-\eta \zeta \hat{J}_{3}^{e}-\partial_{3}\left[\mathrm{i} k_{\alpha} \hat{J}_{\alpha}^{e}-\partial_{3} \hat{J}_{3}^{e}\right]\right. \\
& \left.+\eta \epsilon_{3 \alpha \beta} \mathrm{i} k_{\alpha} \hat{J}_{\beta}^{m}\right) \tilde{G}\left(x_{3}-x_{3}^{S}\right)
\end{aligned}
$$

Working out the derivatives gives

$$
\tilde{E}_{3}=X_{3}^{\mathrm{TM}} \tilde{G}\left(x_{3}-x_{3}^{S}\right)-\frac{\tilde{J}_{3}^{e}}{\eta^{(v)}},
$$

with the source factor given by 


$$
\begin{aligned}
X_{3}^{\mathrm{TM}}= & \frac{\Gamma}{\eta^{(v)}} \operatorname{sign}\left(x_{3}-x_{3}^{S}\right) \mathrm{i} k_{\alpha} \hat{J}_{\alpha}^{e}+\frac{\eta \kappa^{2}}{\left(\eta^{(v)}\right)^{2}} \hat{J}_{3}^{e} \\
& +\frac{\eta}{\eta^{(v)}} \epsilon_{3 \alpha \beta} \mathrm{i} k_{\alpha} \hat{J}_{\beta}^{m},
\end{aligned}
$$

where the sign function is defined as

$$
\operatorname{sign}\left(x_{3}-x_{3}{ }^{\prime}\right)= \begin{cases}-1 & \text { for } x_{3}<x_{3}^{S}, \\ 0 & \text { for } x_{3}=x_{3}^{S} \\ 1 & \text { for } x_{3}>x_{3}^{S}\end{cases}
$$

The vertical component of the magnetic field is found by direct substitution as described above.

To obtain the horizontal electric field components from the vertical electric and magnetic field components, first, both sides of equation 15 are divided by $\eta$ and multiplied by $\mathrm{i} k_{\lambda} \mathrm{i} k_{\alpha}$

$$
\mathrm{i} k_{\lambda} \mathrm{i} k_{\alpha} \tilde{E}_{\alpha}=-\eta^{-1} \mathrm{i} k_{\lambda}\left[\mathrm{i} k_{\alpha} \tilde{J}_{\alpha}^{e}-\mathrm{i} k_{\alpha} \epsilon_{\alpha 3 \beta} \partial_{3} \tilde{H}_{\beta}\right],
$$

and then equation 13 is used to eliminate the horizontal components of the magnetic field

$$
\mathrm{i} k_{\lambda} \mathrm{i} k_{\alpha} \tilde{E}_{\alpha}=-\eta^{-1} \mathrm{i} k_{\lambda}\left[\mathrm{i} k_{\alpha} \tilde{J}_{\alpha}^{e}-\partial_{3}\left(\eta^{(v)} \tilde{E}_{3}+\tilde{J}_{3}^{e}\right)\right] .
$$

Then, both sides of equation 14 are multiplied by $\epsilon_{\alpha \beta 3} \mathrm{i} k_{\beta}$ to obtain

$$
\mathrm{i} k_{\alpha} \mathrm{i} k_{\lambda} \tilde{E}_{\lambda}-\mathrm{i} k_{\lambda} \mathrm{i} k_{\lambda} \tilde{E}_{\alpha}=\epsilon_{\alpha \beta 3} \mathrm{i} k_{\beta}\left(\zeta^{(v)} \tilde{H}_{3}+\tilde{J}_{3}^{m}\right) \text {. }
$$

Next, equation 28 is used to eliminate $\mathrm{i} k_{\alpha} \mathrm{i} k_{\lambda} \tilde{E}_{\lambda}$ from equation 29:

$$
\begin{aligned}
\tilde{E}_{\alpha}= & \frac{\mathrm{i} k_{\alpha}}{\eta \kappa^{2}}\left[\mathrm{i} k_{\beta} \tilde{J}_{\beta}^{e}-\partial_{3}\left(\eta^{(v)} \tilde{E}_{3}+\tilde{J}_{3}^{e}\right)\right] \\
& +\epsilon_{\alpha \beta 3} \frac{\mathrm{i} k_{\beta}}{\kappa^{2}}\left(\zeta^{(v)} \tilde{H}_{3}+\tilde{J}_{3}^{m}\right) .
\end{aligned}
$$

In equation 30, the TM mode and the TE mode are separated from each other because $\tilde{E}_{3}$ is a pure TM mode and $\tilde{H}_{3}$ is a pure TE mode. With the aid of equations 25 and 26, and the corresponding equations for the vertical component of the magnetic field that are obtained from the duality principle, the horizontal electric field components of equation 30 can be written as

$$
\tilde{E}_{\alpha}=X_{\alpha}^{\mathrm{ETM}} \tilde{G}+X_{\alpha}^{\mathrm{ETE}} \tilde{\bar{G}}
$$

where the TE mode Green's function is given by

$$
\tilde{\bar{G}}\left(x_{3}-x_{3}^{S}\right)=\frac{\exp (-\bar{\Gamma} h)}{2 \bar{\Gamma}},
$$

with the vertical wavenumber given by $\bar{\Gamma}=\sqrt{\zeta / \zeta^{(v)}} \sqrt{\kappa^{2}+\bar{\gamma}^{2}}$. The electric-field-related TM mode and TE mode horizontal source components are given by

$$
\begin{aligned}
X_{\alpha}^{\mathrm{ETM}}= & \frac{\mathrm{i} k_{\alpha} \mathrm{i} k_{\beta} \Gamma^{2} \hat{J}_{\beta}^{e}}{\eta \kappa^{2}}+\frac{\mathrm{i} k_{\alpha} \Gamma \hat{J}_{3}^{e} \operatorname{sign}\left(x_{3}-x_{3}^{S}\right)}{\eta^{(v)}} \\
& +\frac{\mathrm{i} k_{\alpha} \epsilon_{3 \lambda \beta} \mathrm{i} k_{\lambda} \Gamma \hat{J}_{\beta}^{m} \operatorname{sign}\left(x_{3}-x_{3}^{S}\right)}{\kappa^{2}},
\end{aligned}
$$

$$
\begin{aligned}
X_{\alpha}^{\mathrm{ETE}}= & -\zeta \frac{\mathrm{i} k_{\alpha} \mathrm{i} k_{\beta} \hat{J}_{\beta}^{e}+\kappa^{2} \hat{J}_{\alpha}^{e}}{\kappa^{2}}+\frac{\epsilon_{\alpha \lambda 3} \mathrm{i} k_{\lambda} \mathrm{i} k_{\beta} \bar{\Gamma} \hat{J}_{\beta}^{m} \operatorname{sign}\left(x_{3}-x_{3}^{S}\right)}{\kappa^{2}} \\
& +\frac{\zeta}{\zeta^{(v)}} \epsilon_{\alpha \beta 3} \mathrm{i} k_{\beta} \hat{J}_{3}^{m} .
\end{aligned}
$$

With the above results the Green's tensors are found as

$$
\begin{aligned}
\tilde{G}_{k r}^{e e}= & \left(\begin{array}{ccc}
\frac{\left(\mathrm{i} k_{1}\right)^{2} \Gamma^{2}}{\eta \kappa^{2}} & \frac{\mathrm{i} k_{1} \mathrm{i} k_{2} \Gamma^{2}}{\eta \kappa^{2}} & -\frac{\mathrm{i} k_{1} \partial_{3}}{\eta^{(v)}} \\
\frac{\mathrm{i} k_{1} \mathrm{i} k_{2} \Gamma^{2}}{\eta \kappa^{2}} & \frac{\left(\mathrm{i} k_{2}\right)^{2} \Gamma^{2}}{\eta \kappa^{2}} & -\frac{\mathrm{i} k_{2} \partial_{3}}{\eta^{(v)}} \\
-\frac{\mathrm{i} k_{1} \partial_{3}}{\eta^{(v)}} & -\frac{\mathrm{i} k_{2} \partial_{3}}{\eta^{(v)}} & \frac{\partial_{3} \partial_{3}-\eta \zeta}{\eta^{(v)}}
\end{array}\right) \tilde{G} \\
& +\left(\begin{array}{ccc}
\frac{\zeta\left(\mathrm{i} k_{2}\right)^{2}}{\kappa^{2}} & -\frac{\zeta \mathrm{i} k_{1} k_{2}}{\kappa^{2}} & 0 \\
-\frac{\zeta \mathrm{i} k_{1} \mathrm{i} k_{2}}{\kappa^{2}} & \frac{\zeta\left(\mathrm{i} k_{1}\right)^{2}}{\kappa^{2}} & 0 \\
0 & 0 & 0
\end{array}\right) \tilde{\bar{G}}, \\
\tilde{G}_{k r}^{e m}= & \left(\begin{array}{ccc}
\frac{\mathrm{i} k_{1} \mathrm{i} k_{2} \partial_{3}}{\kappa^{2}} & -\frac{\left(\mathrm{i} k_{1}\right)^{2} \partial_{3}}{\kappa^{2}} & 0 \\
\frac{\left(\mathrm{i} k_{2}\right)^{2} \partial_{3}}{\kappa^{2}} & -\frac{\mathrm{i} k_{1} \mathrm{i} k_{2} \partial_{3}}{\kappa^{2}} & 0 \\
-\frac{\eta \mathrm{i} k_{2}}{\eta^{(v)}} & \frac{\eta \mathrm{i} k_{1}}{\eta^{(v)}} & 0
\end{array}\right) \tilde{G} \\
+ & \left(\begin{array}{ccc}
-\frac{\mathrm{i} k_{1} i k_{2} \partial_{3}}{\kappa^{2}} & -\frac{\left(\mathrm{i} k_{2}\right)^{2} \partial_{3}}{\kappa^{2}} & \frac{\zeta \mathrm{i} k_{2}}{\zeta^{(v)}} \\
\frac{\left(\mathrm{i} k_{1}\right)^{2} \partial_{3}}{\kappa^{2}} & \frac{\mathrm{i} k_{1} \mathrm{i} k_{2} \partial_{3}}{\kappa^{2}} & -\frac{\zeta \mathrm{i} k_{1}}{\zeta^{(v)}} \\
0 & 0 & 0
\end{array}\right) \tilde{\bar{G}} .
\end{aligned}
$$

This decomposition demonstrates that once the vertical electric and magnetic field components are known, the entire electromagnetic field is known. From the duality principle, it is clear that if the vertical electric field is known, the vertical magnetic field is also known. The same applies to the horizontal components of the magnetic fields. Hence, finding the solution for the vertical electric field in the layered earth model suffices to know the entire electromagnetic field.

\section{THE SPACE FREQUENCY DOMAIN HOMOGENEOUS SPACE GREEN'S FUNCTIONS}

The elements of equations 35 and 36 correspond to homogeneous space Green's functions and can be transformed back to space-frequency in closed form. This is done in this section and results in similar expressions as, for example, found by Weiglhofer (1990) or Abubakar and Habashy (2006).

In the first element of $\tilde{G}_{\alpha \beta}^{e e}$, the factor $\Gamma /\left(\eta \kappa^{2}\right)$ is rewritten as

$$
\frac{\Gamma}{\eta \kappa^{2}}=\frac{1}{\eta^{(v)} \Gamma}+\frac{\zeta}{\kappa^{2} \Gamma} .
$$

With this substitution the Green's function corresponding to the electric field generated by an electric current source in space domain can be written as an inverse spatial Fourier-Bessel transformation 


$$
\begin{aligned}
& \hat{G}_{\alpha \beta}^{e e}\left(\mathbf{x}, \mathbf{x}^{S}, \omega\right) \\
& \quad=\frac{\partial_{\alpha} \partial_{\beta}}{4 \pi \eta^{(v)}} \int_{\kappa=0}^{\infty} \frac{\exp (-\Gamma h)}{\Gamma} \mathrm{J}_{0}(\kappa r) \kappa \mathrm{d} \kappa \\
& \quad+\frac{\zeta \partial_{\alpha} \partial_{\beta}}{4 \pi} \int_{\kappa=0}^{\infty}\left(\frac{\exp (-\Gamma h)}{\Gamma}-\frac{\exp (-\bar{\Gamma} h)}{\bar{\Gamma}}\right) \mathrm{J}_{0}(\kappa r) \kappa^{-1} \mathrm{~d} \kappa \\
& \quad-\frac{\zeta \delta_{\alpha \beta}}{4 \pi} \int_{\kappa=0}^{\infty} \frac{\exp (-\bar{\Gamma} h)}{\bar{\Gamma}} \mathrm{J}_{0}(\kappa r) \kappa \mathrm{d} \kappa
\end{aligned}
$$

where $r=\sqrt{x^{2}+y^{2}}$ is the horizontal distance between source and receiver and $\mathrm{J}_{0}$ denotes the zero-order Bessel function. The first and third integrals are standard Fourier-Bessel transforms, and the second can be evaluated by first performing one derivative on the Bessel function in the integrands. That leads to

$$
\hat{G}\left(\mathbf{x}, \mathbf{x}^{S}\right)=\frac{1}{4 \pi} \int_{\kappa=0}^{\infty} \frac{\exp (-\Gamma h)}{\Gamma} \mathrm{J}_{0}(\kappa r) \kappa \mathrm{d} \kappa=\frac{\exp (-\gamma R)}{4 \pi R \sqrt{\eta / \eta^{(v)}}},
$$

$$
\hat{\bar{G}}\left(\mathbf{x}, \mathbf{x}^{S}\right)=\frac{1}{4 \pi} \int_{\kappa=0}^{\infty} \frac{\exp (-\bar{\Gamma} h)}{\bar{\Gamma}} \mathrm{J}_{0}(\kappa r) \kappa \mathrm{d} \kappa=\frac{\exp (-\bar{\gamma} \bar{R})}{4 \pi \bar{R} \sqrt{\zeta / \zeta^{(v)}}},
$$

$\partial_{\beta} \int_{\kappa=0}^{\infty} \frac{\exp (-\Gamma h)}{\Gamma} \mathrm{J}_{0}(\kappa r) \kappa^{-1} \mathrm{~d} \kappa=-\frac{x_{\beta}}{r} \int_{\kappa=0}^{\infty} \frac{\exp (-\Gamma h)}{\Gamma} \mathrm{J}_{1}(\kappa r) \mathrm{d} \kappa$,

where the scaled source-receiver distances are given by $R=$ $\sqrt{r^{2}+\eta h^{2} / \eta^{(v)}}, \bar{R}=\sqrt{r^{2}+\zeta h^{2} / \zeta^{(v)}}$. The resulting integral is a standard integral, see Gradshteyn and Ryzhik (1996) (p. 1098, formula 6.637 1.), and can be written as (see Abramowitz and Stegun, 1972)

$$
\begin{aligned}
\int_{\kappa}=0 & \frac{\exp \left(-\sqrt{\kappa^{2}+\alpha^{2}} h\right)}{\sqrt{\kappa^{2}+\alpha^{2}}} \mathrm{~J}_{1}(\kappa r) \mathrm{d} \kappa \\
= & \frac{\exp (-\alpha h)-\exp \left(-\alpha \sqrt{r^{2}+h^{2}}\right)}{\alpha r} .
\end{aligned}
$$

With these results, the horizontal components of the incident electric field can be written as

$$
\begin{gathered}
\hat{G}_{\alpha \beta}^{e e}\left(\mathbf{x}, \mathbf{x}^{S}, s\right)=\frac{\partial_{\alpha} \partial_{\beta}}{\eta^{(v)}} \hat{G}\left(\mathbf{x}, \mathbf{x}^{S}, s\right)-\zeta \delta_{\alpha \beta} \hat{\bar{G}}\left(\mathbf{x}, \mathbf{x}^{S}, s\right) \\
+\frac{\sqrt{\zeta}}{4 \pi \sqrt{\eta}} \partial_{\alpha}\left(\frac{x_{\beta}}{r^{2}}[\exp (-\gamma R)-\exp (-\bar{\gamma} \bar{R})]\right) .
\end{gathered}
$$

The vertical field components are written as

$$
\hat{G}_{3 k}^{e e}\left(\mathbf{x}, \mathbf{x}^{S}, s\right)=\frac{\partial_{3} \partial_{k}-\eta \zeta \delta_{3 k}}{\eta^{(v)}} \hat{G}\left(\mathbf{x}, \mathbf{x}^{S}, s\right) .
$$

Carrying out the derivatives results in

$$
\begin{aligned}
\hat{G}_{\alpha \beta}^{e e}\left(\mathbf{x}, \mathbf{x}^{S}\right)= & {\left[\left(3 \frac{x_{\alpha} x_{\beta}}{R^{2}}-\delta_{\alpha \beta}\right)\left(\frac{1}{\eta^{(v)} R^{2}}+\frac{\gamma}{\eta^{(v)} R}\right)+\zeta \frac{x_{\alpha} x_{\beta}}{R^{2}}\right] \hat{G}\left(\mathbf{x}, \mathbf{x}^{S}\right) } \\
& -\zeta \delta_{\alpha \beta} \hat{\bar{G}}\left(\mathbf{x}, \mathbf{x}^{S}\right)-\zeta \frac{x_{\alpha} x_{\beta}}{r^{2}}\left[\hat{G}\left(\mathbf{x}, \mathbf{x}^{S}\right)-\hat{\bar{G}}\left(\mathbf{x}, \mathbf{x}^{S}\right)\right] \\
& -\sqrt{\zeta}\left(2 \frac{x_{\alpha} x_{\beta}}{r^{2}}-\delta_{\alpha \beta}\right)\left(\frac{\exp (-\gamma R)-\exp (-\bar{\gamma} \bar{R})}{4 \pi \sqrt{\eta} r^{2}}\right),
\end{aligned}
$$

where it can be seen that the last two terms vanish in isotropic media. When $h \neq 0$ the Green's function is a regular function of depth in the limit of zero horizontal source-receiver distance, which is obtained when the first three terms of the Taylor expansion of the exponential functions are taken for $r \downarrow 0$ in the terms that are divided by $r^{2}$. This results in

$$
-\lim _{r \downarrow 0} \frac{\exp (-\gamma R)-\exp (-\bar{\gamma} \bar{R})}{4 \pi \sqrt{\eta \zeta} r^{2}}=\left(\frac{\eta^{(v)}}{\eta}-\frac{\zeta^{(v)}}{\zeta}\right) \frac{\exp (-\sqrt{\eta \zeta} h)}{8 \pi h},
$$

and the Green's function is given by

$$
\begin{aligned}
\hat{G}_{\alpha \beta}^{e e}\left(0,0, x_{3}, x_{3}^{S}\right)= & -\delta_{\alpha \beta}\left(\left(\frac{\eta^{(v)}}{\eta h^{2}}+\frac{\gamma}{h} \sqrt{\frac{\eta^{(v)}}{\eta}}\right) \frac{1}{\eta}\right. \\
& \left.+\frac{\zeta^{(v)}}{2}+\frac{\gamma^{2}}{2 \eta}\right) \frac{\exp (-\sqrt{\eta \zeta} h)}{4 \pi h} .
\end{aligned}
$$

The vertical components are given by

$$
\begin{aligned}
\hat{G}_{3 \alpha}^{e e}\left(\mathbf{x}, \mathbf{x}^{S}\right)= & \frac{\eta\left(x_{3}-x_{3}^{S}\right) x_{\alpha}}{\eta^{(v)} R^{2}}\left(\frac{3}{R^{2}}+\frac{3 \gamma}{R}+\gamma^{2}\right) \frac{\hat{G}\left(\mathbf{x}, \mathbf{x}^{S}, s\right)}{\eta^{(v)}}, \\
\hat{G}_{33}^{e e}\left(\mathbf{x}, \mathbf{x}^{S}\right)= & {\left[\left(3\left(\frac{\eta\left(x_{3}-x_{3}^{S}\right)}{\eta^{(v)} R}\right)^{2}-\frac{\eta}{\eta^{(v)}}\right)\left(\frac{1}{R^{2}}+\frac{\gamma}{R}\right)\right.} \\
& \left.+\left(\frac{\left(x_{3}-x_{3}^{S}\right)^{2} \eta}{R^{2} \eta^{(v)}}-1\right) \eta \zeta\right] \frac{\hat{G}\left(\mathbf{x}, \mathbf{x}^{S}\right)}{\eta^{(v)}}
\end{aligned}
$$

Note that the latter equation is only valid for $\mathbf{x} \neq \mathbf{x}^{S}$ if $h=0$. With these results, the solutions for the components of the electric field generated by magnetic point sources are readily obtained as

$$
\begin{aligned}
\hat{G}_{\alpha \beta}^{e m}\left(\mathbf{x}, \mathbf{x}^{S}, s\right)= & \frac{\partial_{3} \epsilon_{\beta \nu 3} \partial_{\nu}}{4 \pi \sqrt{\eta \zeta}} \frac{x_{\alpha}}{r^{2}} \exp (-\gamma R) \\
& -\frac{\partial_{3} \epsilon_{\alpha \nu 3} \partial_{\nu}}{4 \pi \sqrt{\eta \zeta}} \frac{x_{\beta}}{r^{2}} \exp (-\bar{\gamma} \bar{R}), \\
\hat{G}_{\alpha 3}^{e m}\left(\mathbf{x}, \mathbf{x}^{S}, s\right)= & \frac{\zeta}{\zeta^{(v)}} \epsilon_{\alpha \nu 3} \partial_{\nu} \hat{\bar{G}}\left(\mathbf{x}, \mathbf{x}^{S}, s\right),
\end{aligned}
$$

$$
\hat{G}_{3 \beta}^{e m}\left(\mathbf{x}, \mathbf{x}^{S}, s\right)=-\frac{\eta}{\eta^{(v)}} \epsilon_{\beta \iota 3} \partial_{\nu} \hat{G}\left(\mathbf{x}, \mathbf{x}^{S}, s\right),
$$

$$
\hat{G}_{33}^{e m}\left(\mathbf{x}, \mathbf{x}^{S}, s\right)=0
$$




\section{THE LAYERED EARTH RESPONSE}

Because we use lowercase Latin subscripts to denote layer numbers, the subscript notation is abandoned and replaced by vector notation in this section, to avoid confusion. The depth-coordinate in space is now denoted as $z=x_{3}$. First, the layered earth model is built. The upper half-space is a homogeneous medium occupying the domain $\mathbb{D}_{0}$, which is the half-space defined by $z<0$. Every layer of finite thickness occupies a domain $\mathbb{D}_{n}$ and is defined by $z_{n-1}<z<z_{n}$, for $n=1,2,3, \ldots, N$. The lower half-space occupies the domain $\mathbb{D}_{N+1}$ defined by $z>z_{N}$. In layer $\mathbb{D}_{n}$, the medium parameters are constant and given by $\eta_{n}, \eta_{n}^{(v)}, \zeta_{n}, \zeta_{n}^{(v)}$. The source is located in layer $\mathbb{D}_{s}$. The vertical component of the electric field can be written as

$$
\begin{aligned}
\tilde{E}_{z ; n}= & A_{n}^{+} \exp \left[-\Gamma_{n}\left(z-z_{n-1}\right)\right] \\
& +A_{n}^{-} \exp \left[-\Gamma_{n}\left(z_{n}-z\right)\right], \quad \text { for } 0 \leq n<s, \\
\tilde{E}_{z ; s}= & X^{\mathrm{TM}} \exp \left(-\Gamma_{s}\left|z-z^{\prime}\right|\right)-\frac{\hat{J}_{z}^{e}}{\eta_{s}^{(v)}} \delta\left(z-z^{\prime}\right) \\
& +A_{s}^{+} \exp \left[-\Gamma_{s}\left(z-z_{s-1}\right)\right]+A_{s}^{-} \exp \left[-\Gamma_{s}\left(z_{s}-z\right)\right],
\end{aligned}
$$

$$
\begin{aligned}
\tilde{E}_{z ; n}= & A_{n}^{+} \exp \left[-\Gamma_{n}\left(z-z_{n-1}\right)\right] \\
& +A_{n}^{-} \exp \left[-\Gamma_{n}\left(z_{n}-z\right)\right], \quad \text { for } s<n \leq N+1,
\end{aligned}
$$

where $A_{n}^{ \pm}$denote down-/upgoing electric fields. Then, $A_{0}^{+}=0$ and $A_{N+1}^{-}=0$ because there are only upgoing fields in the upper halfspace and only downgoing fields in the lower half-space. The source factor is $X^{\mathrm{TM}}=X_{3}^{\mathrm{TM}} /(2 \Gamma)$. For all layers $\mathbb{D}_{0}$ to $\mathbb{D}_{s-1}$, an up-down global reflection coefficient can be defined as

$$
R_{n}^{-}=\frac{A_{n}^{+}}{A_{n}^{-}} \exp \left(\Gamma_{n} d_{n}\right)
$$

Note that the reflection coefficient $R_{n}^{-}$is defined at depth level $z_{n-1}$. Hence, $R_{1}^{-}$is the global reflection coefficient at the reflector at depth level $z_{0}$. For all layers $\mathbb{D}_{s+1}$ to $\mathbb{D}_{N+1}$, a down-up global reflection coefficient can be defined as

$$
R_{n}^{+}=\frac{A_{n}^{-}}{A_{n}^{+}} \exp \left(\Gamma_{n} d_{n}\right)
$$

The reflection coefficient $R_{n}^{+}$is defined at depth level $z_{n}$. Hence, both global reflection coefficients have the subscript that belongs to the domain where they are defined. In these expressions the thickness of layer $n$ is denoted as $d_{n}=z_{n}-z_{n-1}$. This means the vertical component of the electric field can be written as

$\tilde{E}_{z ; n}=A_{n}^{-}\left(\exp \left[-\Gamma_{n}\left(z_{n}-z\right)\right]+R_{n}^{-} \exp \left[-\Gamma_{n}\left(d_{n}+z-z_{n-1}\right)\right]\right)$

for $0 \leq n<s$, and $R_{0}^{-}=0$. For layers below the source layer, $s<n \leq N+1$, the vertical electric field component can be written as

$$
\tilde{E}_{z ; n}=A_{n}^{+}\left(\exp \left[-\Gamma_{n}\left(z-z_{n-1}\right)\right]+R_{n}^{+} \exp \left[-\Gamma_{n}\left(d_{n}+z_{n}-z\right)\right]\right),
$$

with $R_{N+1}^{+}=0$. Note that with the definitions of the global reflection coefficients, the down- and upgoing electric fields are zerophase at the boundary where they are generated. This leads in a natural way to numerically stable algorithms.

The TM-mode source factor as given in equation 26 is signed for horizontal electric dipole sources, whereas it is unsigned for all other sources. This requires some care in solving for the up- and downgoing field amplitudes in the source layer. To solve for these two amplitude coefficients in the source layer, two boundary conditions are required at interfaces $z_{s-1}$ and $z_{s}$. With two suitable conditions, we can solve this scalar problem. We use the continuity of the vertical electric current and the continuity of the scaled vertical derivative of the vertical component of the electric current. The latter condition can be obtained from equation 30 because the horizontal components of the electric field should be continuous across an interface. These conditions can be written as

$$
\begin{aligned}
\lim _{z \uparrow z_{n}} \eta_{n-1}^{(v)} \tilde{E}_{z ; n-1}\left(\mathbf{k}_{T}, z, s\right) & =\lim _{z \downarrow z_{n}} \eta_{n}^{(v)} \tilde{E}_{z ; n}\left(\mathbf{k}_{T}, z, s\right), \\
\lim _{z \uparrow z_{n}} \frac{\eta_{n-1}^{(v)}}{\eta_{n-1}} \partial_{z} \tilde{E}_{z ; n-1}\left(\mathbf{k}_{T}, z, s\right) & =\lim _{z \downarrow z_{n}} \frac{\eta_{n}^{(v)}}{\eta_{n}} \partial_{z} \tilde{E}_{z ; n}\left(\mathbf{k}_{T}, z, s\right) .
\end{aligned}
$$

At $z=z_{s-1}$ the conditions allow us to eliminate the amplitude $A_{s-1}^{-}$ to obtain

$A_{s}^{+}=R_{s}^{-}\left(X^{\mathrm{TM}}\left(z_{s-1}\right) \exp \left[-\Gamma_{s}\left(z^{\prime}-z_{s-1}\right)\right]+A_{s}^{-} \exp \left(-\Gamma_{s} d_{s}\right)\right)$.

At $z=z_{s}$ the conditions allow us to eliminate the amplitude $A_{s+1}^{+}$to obtain

$$
A_{s}^{-}=R_{s}^{+}\left(X^{\mathrm{TM}}\left(z_{s}\right) \exp \left[-\Gamma_{s}\left(z_{s}-z^{\prime}\right)\right]+A_{s}^{+} \exp \left(-\Gamma_{s} d_{s}\right)\right) .
$$

In these expressions, the global reflection coefficients $R_{n}^{ \pm}$are recursively defined as

$$
R_{n}^{ \pm}=\frac{r_{n}^{ \pm}+R_{n \pm 1}^{ \pm} \exp \left(-2 \Gamma_{n \pm 1} d_{n \pm 1}\right)}{1+r_{n}^{ \pm} R_{n \pm 1}^{ \pm} \exp \left(-2 \Gamma_{n \pm 1} d_{n \pm 1}\right)},
$$

with local reflection coefficients

$$
r_{n}^{ \pm}=\frac{\eta_{n \pm 1} \Gamma_{n}-\eta_{n} \Gamma_{n \pm 1}}{\eta_{n \pm 1} \Gamma_{n}+\eta_{n} \Gamma_{n \pm 1}}
$$

Note that the global reflection coefficient $R_{n}^{-}$contains reflection information about the layers at and above $z_{n-1}$, and the global reflection coefficient $R_{n}^{+}$contains information about the layers at and below $z_{n}$. In contrast, the local reflection coefficients only contain information about one layer. Equations 62 and 63 can be solved for the up- and downgoing field amplitudes in the source layer. They are given by 
$A_{s}^{-}=\frac{R_{s}^{+}\left(X^{\mathrm{TM}}\left(z_{s}\right) \exp \left(-\Gamma_{s} d^{+}\right)+R_{s}^{-} X^{\mathrm{TM}}\left(z_{s-1}\right) \exp \left[-\Gamma_{s}\left(d_{s}+d^{-}\right)\right]\right)}{1-R_{s}^{-} R_{s}^{+} \exp \left(-2 \Gamma_{s} d_{s}\right)}$,

$A_{s}^{+}=\frac{R_{s}^{-}\left(X^{\mathrm{TM}}\left(z_{s-1}\right) \exp \left(-\Gamma_{s} d^{-}\right)+R_{s}^{+} X^{\mathrm{TM}}\left(z_{s}\right) \exp \left[-\Gamma_{s}\left(d_{s}+d^{+}\right)\right]\right)}{1-R_{s}^{-} R_{s}^{+} \exp \left(-2 \Gamma_{s} d_{s}\right)}$,

where the distances from the interfaces to the source depth are given by $d^{-}=z^{\prime}-z_{s-1}$ and $d^{+}=z_{s}-z^{\prime}$. If the receivers are in the source layer, the electric field is now determined. If the receivers are in any layer above the source layer, the field can be recursively built by using the continuity of vertical electric current across each interface as expressed in equation 60 . The solution for $A_{s-1}^{-}$in the layer just above the source is

$$
\begin{aligned}
A_{s-1}^{-}= & \frac{\eta_{s}^{(v)}}{\eta_{s-1}^{(v)}} \\
& \times \frac{\left\{X^{\mathrm{TM}}\left(z_{s-1}\right) \exp \left(-\Gamma_{s} d^{-}\right)+X^{\mathrm{TM}}\left(z_{s}\right) R_{s}^{+} \exp \left[-\Gamma_{s}\left(d_{s}+d^{+}\right)\right]\right\}\left(1+R_{s}^{-}\right)}{\left[1-R_{s}^{-} R_{s}^{+} \exp \left(-2 \Gamma_{s} d_{s}\right)\right]\left[1+R_{s-1}^{-} \exp \left(-2 \Gamma_{s-1} d_{s-1}\right)\right]} .
\end{aligned}
$$

For all the other layers above the source layer, they can be found recursively as

$$
A_{n}^{-}=\frac{\eta_{n+1}^{(v)}}{\eta_{n}^{(v)}} A_{n+1}^{-} \frac{\left(1+R_{n+1}^{-}\right) \exp \left(-\Gamma_{n+1} d_{n+1}\right)}{1+R_{n}^{-} \exp \left(-2 \Gamma_{n} d_{n}\right)},
$$

for $0 \leq n<s-1$. It can also be written explicitly as

$$
A_{n}^{-}=\frac{\eta_{s-1}^{(v)}}{\eta_{n}^{(v)}} A_{s-1}^{-} \prod_{m=n}^{s-2} \frac{\left(1+R_{m+1}^{-}\right) \exp \left(-\Gamma_{m+1} d_{m+1}\right)}{1+R_{m}^{-} \exp \left(-2 \Gamma_{m} d_{m}\right)},
$$

for $0 \leq n<s-1$. In case the receivers are below the sources, the electric field can be recursively obtained for layers below the source in a similar way. The solution for the layer just below the source is obtained as

$$
\begin{aligned}
A_{s+1}^{+}= & =\frac{\eta_{s}^{(v)}}{\eta_{s+1}^{(v)}} \\
& \times \frac{\left\{X^{\mathrm{TM}}\left(z_{s-1}\right) R_{s}^{-} \exp \left[-\Gamma_{s}\left(d_{s}+d^{-}\right)\right]+X^{\mathrm{TM}}\left(z_{s}\right) \exp \left(-\Gamma_{s} d^{+}\right)\right\}\left(1+R_{s}^{+}\right)}{\left[1-R_{s}^{-} R_{s}^{+} \exp \left(-2 \Gamma_{s} d_{s}\right)\right]\left[1+R_{s+1}^{+} \exp \left(-2 \Gamma_{s+1} d_{s+1}\right)\right]} .
\end{aligned}
$$

For all the other layers below the source layer, they can be found recursively as

$$
A_{n}^{+}=\frac{\eta_{n-1}^{(v)}}{\eta_{n}^{(v)}} A_{n-1}^{+} \frac{\left(1+R_{n-1}^{+}\right) \exp \left(-\Gamma_{n-1} d_{n-1}\right)}{1+R_{n}^{+} \exp \left(-2 \Gamma_{n} d_{n}\right)},
$$

for $s+2 \leq n \leq N+1$. It can also be written explicitly as

$$
A_{n}^{+}=\frac{\eta_{s+1}^{(v)}}{\eta_{n}^{(v)}} A_{s+1}^{+} \prod_{m=s+2}^{n} \frac{\left(1+R_{m-1}^{+}\right) \exp \left(-\Gamma_{m-1} d_{m-1}\right)}{1+R_{m}^{+} \exp \left(-2 \Gamma_{m} d_{m}\right)},
$$

for $s+2 \leq n \leq N+1$. Note that all terms only contain damped exponentials and can be computed numerically without any difficulty.
Now the vertical electric field component is determined everywhere in the model. This solves the electromagnetic problem in layered VTI media. The construction of the full solution is a bookkeeping task. We construct it in the next section using the Green's functions.

\section{THE GREEN'S FUNCTIONS FOR THE VERTICAL ELECTRIC FIELD IN THE FREQUENCY WAVENUMBER DOMAIN}

The expressions for the electromagnetic fields can be written in terms of the Green's functions defined in equations 5-8. Here, they are given in the horizontal wavenumber domain for receivers anywhere in the model. With the substitution of equation 26 divided by $2 \Gamma_{s}$ into the solutions for the amplitude coefficients of equations 66 and 67 in equations 53-55, all Green's function components related to the vertical electric field component can be found. We introduce field propagators inside each layer as

$$
W_{n}^{u}=\exp \left[-\Gamma_{n}\left(z_{n}-z\right)\right], \quad W_{n}^{d}=\exp \left[-\Gamma_{n}\left(z-z_{n-1}\right)\right],
$$

with $z_{n-1} \leq z \leq z_{n}$, for $0 \leq n \leq N+1$.

The vertical electric field Green's function components in the source layer generated by electric point sources are obtained as

$$
\begin{array}{r}
\tilde{G}_{z x ; s}^{e e}=\tilde{G}_{z x ; s}^{e e ; i}+\frac{\mathrm{i} k_{x}}{2 \eta_{s}^{(v)}}\left\{P_{s}^{u-} W_{s}^{u}-P_{s}^{d-} W_{s}^{d}\right\}, \\
\tilde{G}_{z y ; s}^{e e}=\tilde{G}_{z y ; s}^{e e ; i}+\frac{\mathrm{i} k_{y}}{2 \eta_{s}^{(v)}}\left\{P_{s}^{u-} W_{s}^{u}-P_{s}^{d-} W_{s}^{d}\right\}, \\
\tilde{G}_{z z ; s}^{e e}=\tilde{G}_{z z ; s}^{e e ; i}+\frac{\eta_{s} \kappa^{2}}{2\left(\eta_{s}^{(v)}\right)^{2} \Gamma_{s}}\left\{P_{s}^{u+} W_{s}^{u}+P_{s}^{d+} W_{s}^{d}\right\},
\end{array}
$$

where the incident fields and the up- and downgoing fields are written as

$$
\begin{gathered}
\tilde{G}_{z x ; i}^{e e ; i}=-\frac{\mathrm{i} k_{x} \partial_{z}}{\eta_{s}^{(v)}} \frac{\exp \left(-\Gamma_{s}\left|z-z^{\prime}\right|\right)}{2 \Gamma_{s}}, \\
\tilde{G}_{z y ; i}^{e e ; i}=-\frac{\mathrm{i} k_{y} \partial_{z}}{\eta_{s}^{(v)}} \frac{\exp \left(-\Gamma_{s}\left|z-z^{\prime}\right|\right)}{2 \Gamma_{s}}, \\
\tilde{G}_{z z ; s}^{e e ; i}=\frac{-\eta_{s} \zeta_{s}+\partial_{z} \partial_{z}}{\eta_{s}^{(v)}} \frac{\exp \left(-\Gamma_{s}\left|z-z^{\prime}\right|\right)}{2 \Gamma_{s}}, \\
P_{s}^{u \pm}=\frac{R_{s}^{+}}{M_{s}}\left(\exp \left(-\Gamma_{s} d^{+}\right) \pm R_{s}^{-} \exp \left[-\Gamma_{s}\left(d_{s}+d^{-}\right)\right]\right),
\end{gathered}
$$

$$
P_{s}^{d \pm}=\frac{R_{s}^{-}}{M_{s}}\left(\exp \left(-\Gamma_{s} d^{-}\right) \pm R_{s}^{+} \exp \left[-\Gamma_{s}\left(d_{s}+d^{+}\right)\right]\right)
$$

In these equations, the factor $M_{s}$ is given by

$$
M_{s}=1-R_{s}^{-} R_{s}^{+} \exp \left(-2 \Gamma_{s} d_{s}\right) .
$$


The vertical electric field Green's function components in the source layer generated by magnetic point sources are given by

$$
\begin{gathered}
\tilde{G}_{z x ; s}^{e m}=\tilde{G}_{z x ; s}^{e m ; i}-\frac{\eta_{s}^{\mathrm{i} k_{y}}}{2 \eta_{s}^{(v)} \Gamma_{s}}\left\{P_{s}^{u+} W_{s}^{u}+P_{s}^{d+} W_{s}^{d}\right\}, \\
\tilde{G}_{z y ; s}^{e m}=\tilde{G}_{z y ; s}^{e m ; i}+\frac{\eta_{s} \mathrm{i} k_{x}}{2 \eta_{s}^{(v)} \Gamma_{s}}\left\{P_{s}^{u+} W_{s}^{u}+P_{s}^{d+} W_{s}^{d}\right\}, \\
\tilde{G}_{z z ; s}^{e m}=0 .
\end{gathered}
$$

The incident fields are given by

$$
\begin{gathered}
\tilde{G}_{z x ; s}^{e m ; i}=-\frac{\eta_{s} \mathrm{i} k_{y}}{\eta_{s}^{(v)}} \frac{\exp \left(-\Gamma_{s}\left|z-z^{\prime}\right|\right)}{2 \Gamma_{s}} \\
\tilde{G}_{z y ; s}^{e m ; i}=\frac{\eta_{s} \mathrm{i} k_{x}}{\eta_{s}^{(v)}} \frac{\exp \left(-\Gamma_{s}\left|z-z^{\prime}\right|\right)}{2 \Gamma_{s}} .
\end{gathered}
$$

In the layers above the source, we find

$$
\begin{gathered}
\tilde{G}_{z x ; n}^{e e}=\frac{\mathrm{i} k_{x}}{2 \eta_{n}^{(v)}} P_{n}^{u-}\left(W_{n}^{u}+R_{n}^{-} \exp \left(-\Gamma_{n} d_{n}\right) W_{n}^{d}\right), \\
\tilde{G}_{z y ; n}^{e e}=\frac{\mathrm{i} k_{y}}{2 \eta_{n}^{(v)}} P_{n}^{u-}\left(W_{n}^{u}+R_{n}^{-} \exp \left(-\Gamma_{n} d_{n}\right) W_{n}^{d}\right), \\
\tilde{G}_{z z ; n}^{e e}=\frac{\eta_{s} \kappa^{2}}{2 \eta_{s}^{(v)} \eta_{n}^{(v)} \Gamma_{s}} P_{n}^{u+}\left(W_{n}^{u}+R_{n}^{-} \exp \left(-\Gamma_{n} d_{n}\right) W_{n}^{d}\right),
\end{gathered}
$$

and

$$
\begin{gathered}
\tilde{G}_{z x ; n}^{e m}=-\frac{\eta_{s}^{\mathrm{i} k_{y}}}{2 \eta_{n}^{(v)} \Gamma_{s}} P_{n}^{u+}\left(W_{n}^{u}+R_{n}^{-} \exp \left(-\Gamma_{n} d_{n}\right) W_{n}^{d}\right), \\
\tilde{G}_{z y ; n}^{e m}=\frac{\eta_{s} \mathrm{i} k_{x}}{2 \eta_{n}^{(v)} \Gamma_{s}} P_{n}^{u+}\left(W_{n}^{u}+R_{n}^{-} \exp \left(-\Gamma_{n} d_{n}\right) W_{n}^{d}\right), \\
\tilde{G}_{z z ; n}^{e m}=0,
\end{gathered}
$$

for $n=s-1$ and $0 \leq n<s-1$. The factors $P_{n}^{ \pm}$are given by

$$
P_{s-1}^{u \pm}=\frac{\left(1+R_{s}^{-}\right)\left\{ \pm \exp \left(-\Gamma_{s} d^{-}\right)+R_{s}^{+} \exp \left[-\Gamma_{s}\left(d_{s}+d^{+}\right)\right]\right\}}{M_{s}\left[1+R_{s-1}^{-} \exp \left(-2 \Gamma_{s-1} d_{s-1}\right)\right]},
$$

$$
P_{n}^{u \pm}=P_{s-1}^{u \pm} \prod_{m=n}^{s-2} \frac{\left(1+R_{m+1}^{-}\right) \exp \left(-\Gamma_{m+1} d_{m+1}\right)}{1+R_{m}^{-} \exp \left(-2 \Gamma_{m} d_{m}\right)}
$$

In the layers below the source, we find

$$
\begin{gathered}
\tilde{G}_{z x ; n}^{e e}=\frac{\mathrm{i} k_{x}}{2 \eta_{n}^{(v)}} P_{n}^{d-}\left(W_{n}^{d}+R_{n}^{+} \exp \left(-\Gamma_{n} d_{n}\right) W_{n}^{u}\right), \\
\tilde{G}_{z y ; n}^{e e}=\frac{\mathrm{i} k_{y}}{2 \eta_{n}^{(v)}} P_{n}^{d-}\left(W_{n}^{d}+R_{n}^{+} \exp \left(-\Gamma_{n} d_{n}\right) W_{n}^{u}\right), \\
\tilde{G}_{z z ; n}^{e e}=\frac{\eta_{s} \kappa^{2}}{2 \eta_{s}^{(v)} \eta_{n}^{(v)} \Gamma_{s}} P_{n}^{d+}\left(W_{n}^{d}+R_{n}^{+} \exp \left(-\Gamma_{n} d_{n}\right) W_{n}^{u}\right),
\end{gathered}
$$

and

$$
\begin{gathered}
\tilde{G}_{z x ; n}^{e m}=-\frac{\eta_{s} \mathrm{i} k_{y}}{2 \eta_{n}^{(v)} \Gamma_{s}} P_{n}^{d+}\left(W_{n}^{d}+R_{n}^{+} \exp \left(-\Gamma_{n} d_{n}\right) W_{n}^{u}\right), \\
\tilde{G}_{z y ; n}^{e m}=\frac{\eta_{s} \mathrm{i} k_{x}}{2 \eta_{n}^{(v)} \Gamma_{s}} P_{n}^{d+}\left(W_{n}^{d}+R_{n}^{+} \exp \left(-\Gamma_{n} d_{n}\right) W_{n}^{u}\right),
\end{gathered}
$$

$$
\tilde{G}_{z z ; n}^{e m}=0,
$$

for $n=s+1$ and $s+1<n \leq N+1$. The factors $P_{n}^{ \pm}$are given by

$$
\begin{gathered}
P_{s+1}^{d \pm}=\frac{\left(1+R_{s}^{+}\right)\left\{\exp \left(-\Gamma_{s} d^{+}\right) \pm R_{s}^{-} \exp \left[-\Gamma_{s}\left(d_{s}+d^{-}\right)\right]\right\}}{M_{s}\left[1+R_{s+1}^{+} \exp \left(-2 \Gamma_{s+1} d_{s+1}\right)\right]} \\
P_{n}^{d \pm}=P_{s+1}^{d \pm} \prod_{m=s+2}^{n} \frac{\left(1+R_{m-1}^{+}\right) \exp \left(-\Gamma_{m-1} d_{m-1}\right)}{1+R_{m}^{+} \exp \left(-2 \Gamma_{m} d_{m}\right)}
\end{gathered}
$$

With these results, the VTI layered-earth problem has been solved. The expression for the vertical component for the magnetic field, the other required scalar differential equation, can be obtained using the duality principle. Then, the horizontal components of the electric field can be obtained from equation 30, and the horizontal components of the magnetic field follow from duality. We list all results in Appendices A and B.

\section{COMPUTATION OF THE ELECTRIC FIELD IN THE SPACE-FREQUENCY DOMAIN}

Space-frequency-domain results are obtained by inverse spatial Fourier transformation. If the receivers are in the source layer, direct and scattered fields must be computed. The direct field is known in explicit form, whereas the scattered field must be computed numerically. If the receivers are not in the source layer, the whole field must be computed numerically. In the source layer, the components of the electric field generated by an $x$-directed electric dipole can be written in the space-frequency domain as 


$$
\begin{aligned}
\hat{G}_{x x ; s}^{e e}\left(\mathbf{x}, \mathbf{x}^{\prime}, \omega\right)= & \hat{G}_{x x ; s}^{e e ; i}\left(\mathbf{x}-\mathbf{x}^{\prime}, \omega\right) \\
& +\frac{1}{8 \pi} \int_{\kappa=0}^{\infty}\left(\frac{\Gamma_{s} \tilde{g}_{h h ; s}^{t m}}{\eta_{s}}-\frac{\zeta_{s} \tilde{g}_{z z ; s}^{t e}}{\bar{\Gamma}_{s}}\right) \mathbf{J}_{0}(\kappa r) \kappa \mathrm{d} \kappa \\
& -\frac{\cos (2 \phi)}{8 \pi} \int_{\kappa=0}^{\infty}\left(\frac{\Gamma_{s} \tilde{g}_{h h ; s}^{t m}}{\eta_{s}}+\frac{\zeta_{s} \tilde{g}_{z z ; s}^{t e}}{\bar{\Gamma}_{s}}\right) \mathbf{J}_{2}(\kappa r) \kappa \mathrm{d} \kappa,
\end{aligned}
$$

$$
\begin{aligned}
\hat{G}_{y x ; s}^{e e}\left(\mathbf{x}, \mathbf{x}^{\prime}, \omega\right)= & \hat{G}_{y x ; s}^{e e ; i}\left(\mathbf{x}-\mathbf{x}^{\prime}, \omega\right) \\
& -\frac{\sin (2 \phi)}{8 \pi} \int_{\kappa=0}^{\infty}\left(\frac{\Gamma_{s} \tilde{g}_{h h ; s}^{t m}}{\eta_{s}}+\frac{\zeta_{s} \tilde{g}_{z z ; s}^{t e}}{\bar{\Gamma}_{s}}\right) \mathbf{J}_{2}(\kappa r) \kappa \mathrm{d} \kappa,
\end{aligned}
$$

$$
\begin{aligned}
\hat{G}_{z x ; s}^{e e}\left(\mathbf{x}, \mathbf{x}^{\prime}, \omega\right)= & \hat{G}_{z x ; s}^{e e ; i}\left(\mathbf{x}-\mathbf{x}^{\prime}, \omega\right) \\
& +\frac{\cos (\phi)}{4 \pi \eta_{s}^{(v)}} \int_{\kappa=0}^{\infty} \tilde{g}_{z h ; s}^{t m} \mathrm{~J}_{1}(\kappa r) \kappa^{2} \mathrm{~d} \kappa
\end{aligned}
$$

where the integrals containing $\mathrm{J}_{1}, \mathrm{~J}_{2}$ vanish for $r=0$. The coefficients are given by

$$
\begin{aligned}
& \tilde{g}_{h h ; s}^{t m}=P_{s}^{u-} W_{s}^{u}+P_{s}^{d-} W_{s}^{d}, \\
& \tilde{g}_{z z ; s}^{t e}=\bar{P}_{s}^{u+} \bar{W}_{s}^{u}+\bar{P}_{s}^{d+} \bar{W}_{s}^{d}, \\
& \tilde{g}_{z h ; s}^{t m}=P_{s}^{u-} W_{s}^{u}-P_{s}^{d-} W_{s}^{d} .
\end{aligned}
$$

The incident fields have already been determined. The electric field components for a $y$-directed electric dipole can be written as

$$
\begin{gathered}
\hat{G}_{x y ; s}^{e e}\left(\mathbf{x}, \mathbf{x}^{\prime}, \omega\right)=\hat{G}_{y x ; s}^{e e}\left(\mathbf{x}, \mathbf{x}^{\prime}, \omega\right), \\
\hat{G}_{y y ; s}^{e e}\left(\mathbf{x}, \mathbf{x}^{\prime}, \omega\right)= \\
+\frac{1}{8 \pi} \int_{\kappa=0}^{e e ; i}\left(\frac{\Gamma_{s} \tilde{g}_{h h ; s}^{t m}}{\eta_{s}}-\frac{\zeta_{s} \tilde{g}_{z z ; s}^{t e}}{\bar{\Gamma}_{s}}\right) \mathbf{J}_{0}(\kappa r) \kappa \mathrm{d} \kappa \\
+\frac{\cos (2 \phi)}{8 \pi} \int_{\kappa=0}^{\infty}\left(\frac{\Gamma_{s} \tilde{g}_{h h ; s}^{t m}}{\eta_{s}}+\frac{\zeta_{s} \tilde{g}_{z z ; s}^{t e}}{\bar{\Gamma}_{s}}\right) \mathrm{J}_{2}(\kappa r) \kappa \mathrm{d} \kappa, \\
\hat{G}_{z y ; s}^{e e}\left(\mathbf{x}, \mathbf{x}^{\prime}, \omega\right)=\hat{G}_{z y ; s}^{e e ; i}\left(\mathbf{x}-\mathbf{x}^{\prime}, \omega\right) \\
+\frac{\sin (\phi)}{4 \pi \eta_{s}^{(v)}} \int_{\kappa=0}^{\infty} \tilde{g}_{z h ; s}^{t m} \mathrm{~J}_{1}(\kappa r) \kappa^{2} \mathrm{~d} \kappa .
\end{gathered}
$$

The vertical electric field generated by a $z$-directed electric dipole can be written as

$$
\begin{aligned}
\hat{G}_{x z ; s}^{e e}\left(\mathbf{x}, \mathbf{x}^{\prime}, \omega\right)= & \hat{G}_{x z ; s}^{e e ; i}\left(\mathbf{x}-\mathbf{x}^{\prime}, \omega\right) \\
& -\frac{\cos (\phi)}{4 \pi \eta_{s}^{(v)}} \int_{\kappa=0}^{\infty} \tilde{g}_{h z ; s}^{t m} \mathrm{~J}_{1}(\kappa r) \kappa^{2} \mathrm{~d} \kappa
\end{aligned}
$$

$$
\begin{aligned}
\hat{G}_{y z ; s}^{e e}\left(\mathbf{x}, \mathbf{x}^{\prime}, \omega\right)= & \hat{G}_{y z ; s}^{e e ; i}\left(\mathbf{x}-\mathbf{x}^{\prime}, \omega\right) \\
& -\frac{\sin (\phi)}{4 \pi \eta_{s}^{(v)}} \int_{\kappa=0}^{\infty} \tilde{g}_{h z ; s}^{t m} \mathrm{~J}_{1}(\kappa r) \kappa^{2} \mathrm{~d} \kappa, \\
\hat{G}_{z z ; s}^{e e}\left(\mathbf{x}, \mathbf{x}^{\prime}, \omega\right)= & \hat{G}_{z z ; s}^{e e ; i}\left(\mathbf{x}-\mathbf{x}^{\prime}, \omega\right) \\
& +\frac{\eta_{s}}{4 \pi\left(\eta_{s}^{(v)}\right)^{2}} \int_{\kappa=0}^{\infty} \tilde{g}_{z z ; s}^{t m} \mathrm{~J}_{0}(\kappa r) \frac{\kappa^{3}}{\Gamma_{s}} \mathrm{~d} \kappa,
\end{aligned}
$$

with the coefficients given by

$$
\begin{aligned}
& \tilde{g}_{h z ; s}^{t m}=\left(P_{s}^{u+} W_{s}^{u}-P_{s}^{d+} W_{s}^{d}\right), \\
& \tilde{g}_{z z ; s}^{t m}=\left(P_{s}^{u+} W_{s}^{u}+P_{s}^{d+} W_{s}^{d}\right) .
\end{aligned}
$$

With these expressions, all components of the electric field Green's function generated by an electric dipole are determined in the source layer. Five integrals need to be computed in a $2 \mathrm{D}$ plane containing the vertical axis to obtain the field values everywhere in the 3D space.

The electric field generated by the $x$-component of a point source of the magnetic current type is given by

$$
\begin{gathered}
\hat{G}_{x x ; s}^{e m}\left(\mathbf{x}, \mathbf{x}^{\prime}, \omega\right)=\hat{G}_{x x ; s}^{e m ; i}\left(\mathbf{x}-\mathbf{x}^{\prime}, \omega\right) \\
+\frac{\sin (2 \phi)}{4 \pi} \int_{\kappa=0}^{\infty}\left(\tilde{g}_{h z ; s}^{t m}+\tilde{g}_{h h ; s}^{t e}\right) \mathbf{J}_{2}(\kappa r) \kappa \mathrm{d} \kappa, \quad(119) \\
\hat{G}_{y x ; s}^{e m}\left(\mathbf{x}, \mathbf{x}^{\prime}, \omega\right)=\hat{G}_{y x ; s}^{e m ; i}\left(\mathbf{x}-\mathbf{x}^{\prime}, \omega\right)-\frac{1}{8 \pi} \int_{\kappa=0}^{\infty}\left(\tilde{g}_{h z ; s}^{t m}-\tilde{g}_{h h ; s}^{t e}\right) \mathrm{J}_{0}(\kappa r) \kappa \mathrm{d} \kappa \\
-\frac{\cos (2 \phi)}{8 \pi} \int_{\kappa=0}^{\infty}\left(\tilde{g}_{h z ; s}^{t m}+\tilde{g}_{h h ; s}^{t e}\right) \mathrm{J}_{2}(\kappa r) \kappa \mathrm{d} \kappa, \quad(120) \\
\hat{G}_{z x ; s}^{e m}\left(\mathbf{x}, \mathbf{x}^{\prime}, \omega\right)=\hat{G}_{z x ; s}^{e m ; i}\left(\mathbf{x}-\mathbf{x}^{\prime}, \omega\right) \\
\quad-\frac{\eta_{s} \sin (\phi)}{8 \pi \eta_{s}^{(v)}} \int_{\kappa=0}^{\infty} \tilde{g}_{z z ; s}^{t m} \mathrm{~J}_{1}(\kappa r) \frac{\kappa^{2}}{\Gamma_{s}} \mathrm{~d} \kappa, \quad(121)
\end{gathered}
$$

with the new coefficient given by

$$
\tilde{g}_{h h ; s}^{t e}=\bar{P}_{s}^{u-} \bar{W}_{s}^{u}-\bar{P}_{s}^{d-} \bar{W}_{s}^{d} .
$$

The electric field generated by the $y$-component of a point source of the magnetic current type is given by

$$
\begin{aligned}
\hat{G}_{x y ; s}^{e m}\left(\mathbf{x}, \mathbf{x}^{\prime}, \omega\right)= & \hat{G}_{x y ; s}^{e m ; i}\left(\mathbf{x}-\mathbf{x}^{\prime}, \omega\right) \\
& +\frac{1}{8 \pi} \int_{\kappa=0}^{\infty}\left(\tilde{g}_{h z ; s}^{t m}-\tilde{g}_{h h ; s}^{t e}\right) \mathbf{J}_{0}(\kappa r) \kappa \mathrm{d} \kappa \\
& -\frac{\cos (2 \phi)}{8 \pi} \int_{\kappa=0}^{\infty}\left(\tilde{g}_{h z ; s}^{t m}+\tilde{g}_{h h ; s}^{t e}\right) \mathbf{J}_{2}(\kappa r) \kappa \mathrm{d} \kappa,
\end{aligned}
$$


a) Amplitude of $\hat{G}_{r x}^{e e}$ using the half-space code

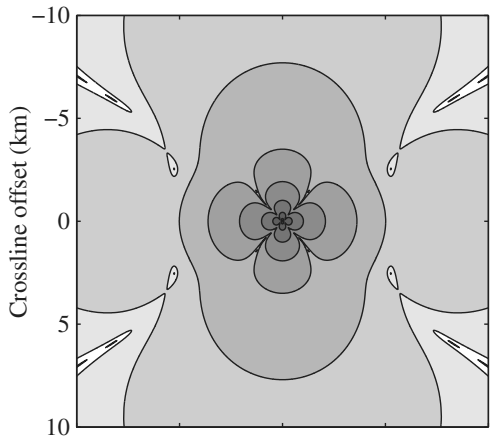

d) Amplitude of $\hat{G}_{v x}^{e e}$ using the half-space code

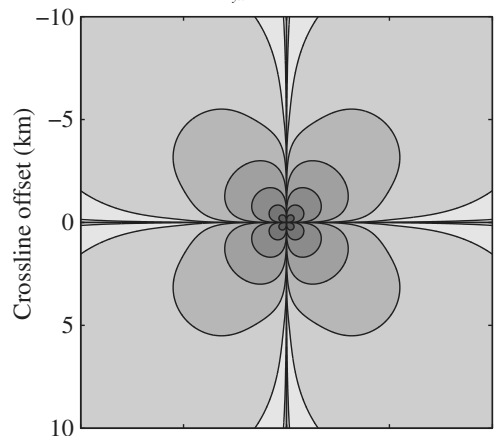

g) Amplitude of $\hat{G}_{z x}^{e e}$ using the half-space code

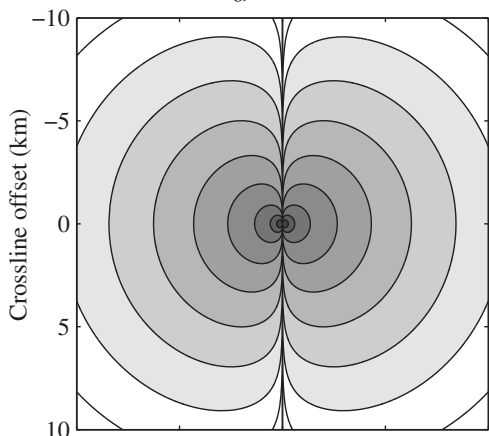

j) Amplitude of $\hat{G}_{z z}^{e e}$ using the half-space code
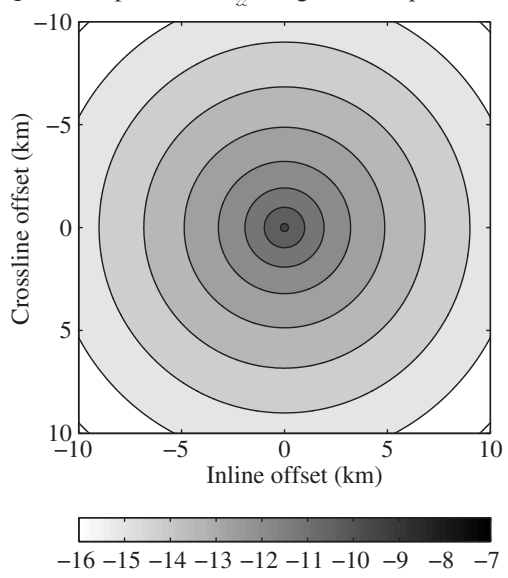

Amplitude $\left(\log _{10}(\mathrm{Vs} / \mathrm{m})\right)$ b)

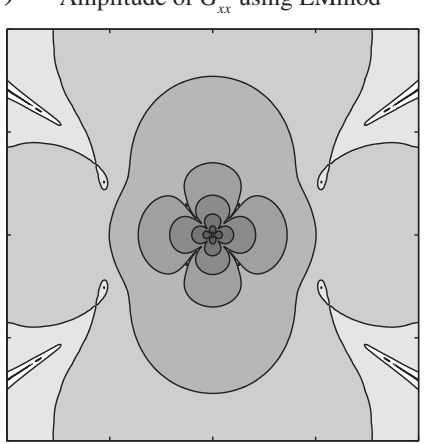

e)

Amplitude of $\hat{G}_{y x}^{e e}$ using EMmod

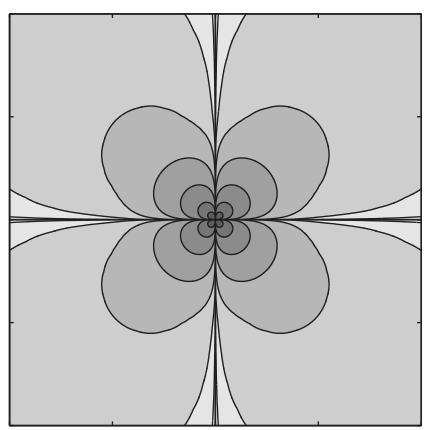

h)

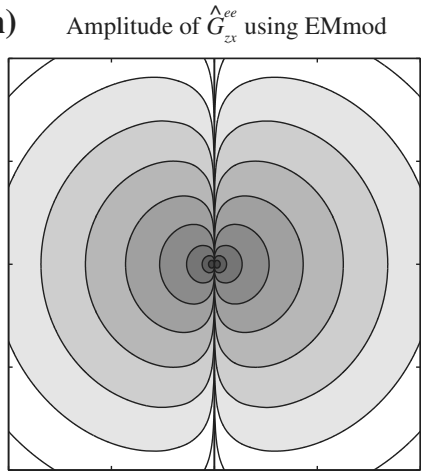

k) Amplitude of $\hat{G}_{z z}^{e e}$ using EMmod
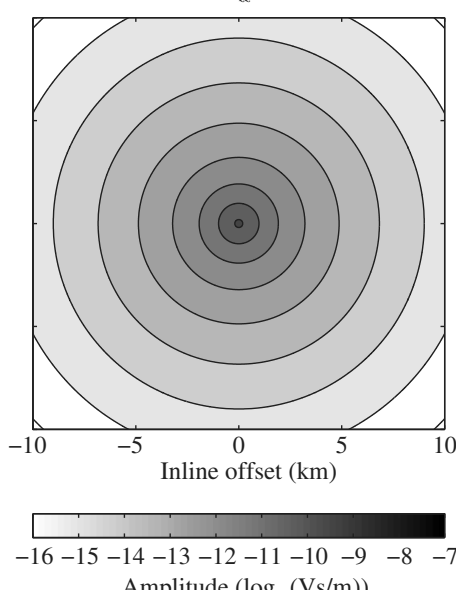

Amplitude $\left(\log _{10}(\mathrm{Vs} / \mathrm{m})\right)$ c) Relative Error $\hat{G}_{x x}^{e e}$

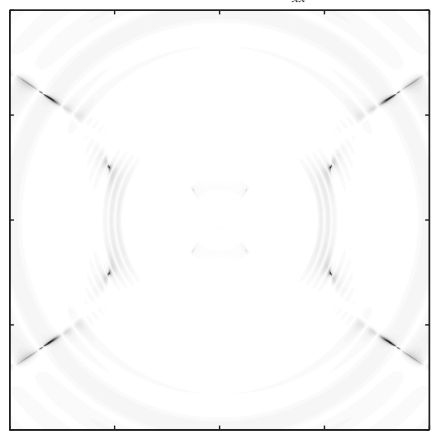

f) Relative Error $\hat{G}_{v x}^{e e}$

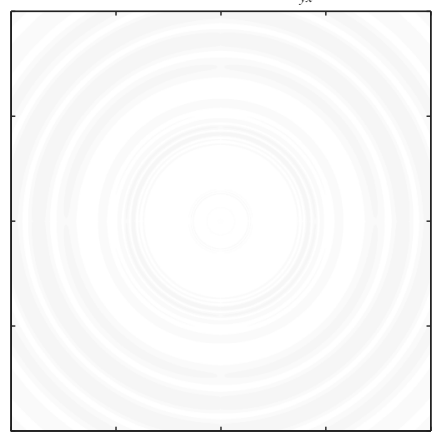

i) Relative Error $\hat{G}_{z x}^{e e}$

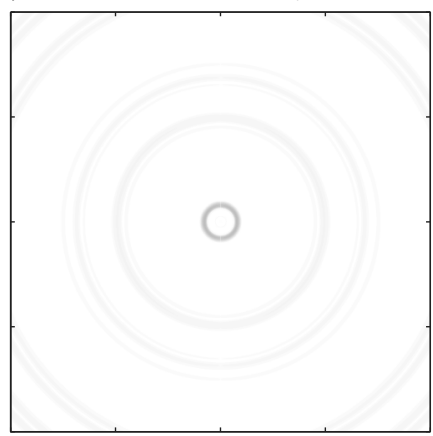

1) Relative Error $\hat{G}_{z z}^{e e}$
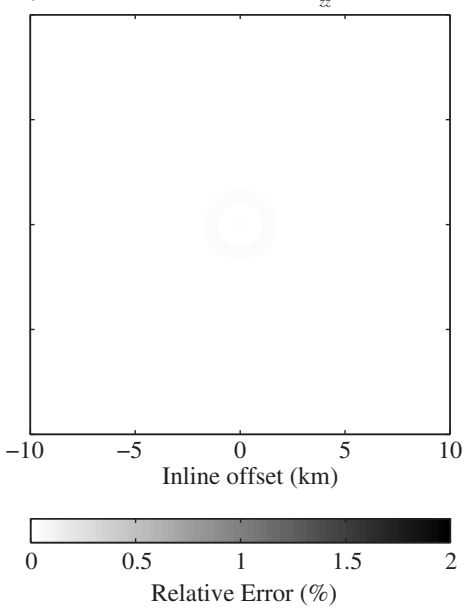

Figure 1. Comparison between the analytical half-space solution and EMmod with respect to the amplitude. Panels (a-c) show the half-space solution, the solution computed with EMmod, and the relative error between the two for electric inline oriented receivers and an electric inline oriented source $\left(\hat{G}_{x x}^{e e}\right)$. Panels (d-f) show the same for $\hat{G}_{y x}^{e e}$, panels $(\mathrm{g}-\mathrm{i})$ for $\hat{G}_{z x}^{e e}$, and panels $(\mathrm{j}-1)$ for $\hat{G}_{z z}^{e e}$. 
a) Phase of $\hat{G}_{x x}^{e e}$ using the half-space code

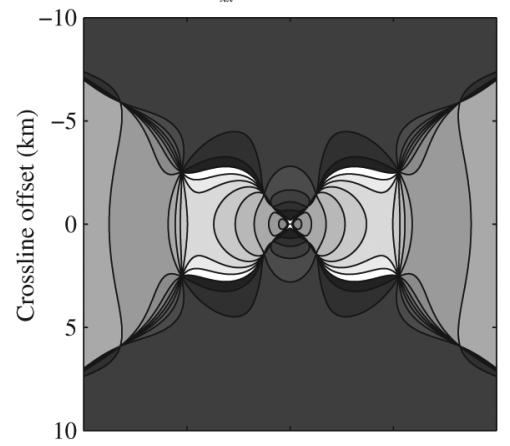

d)

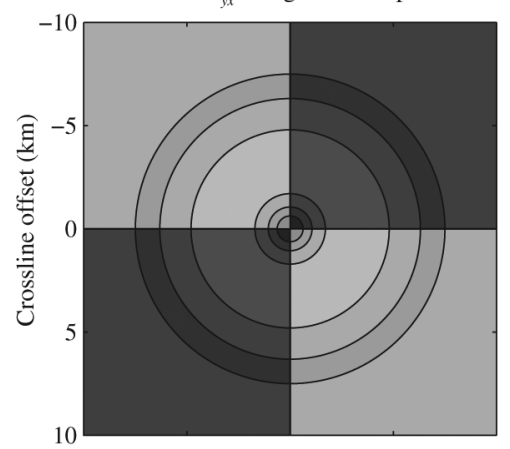

g)

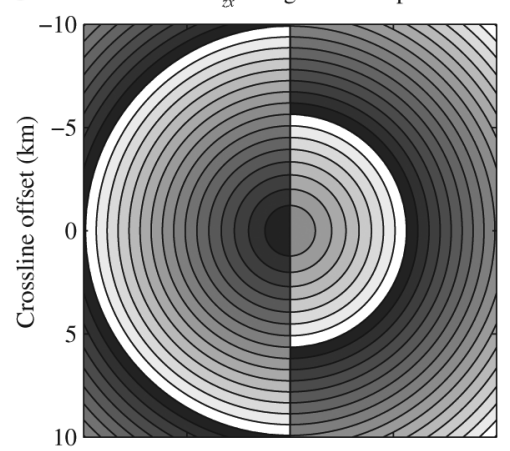

j)

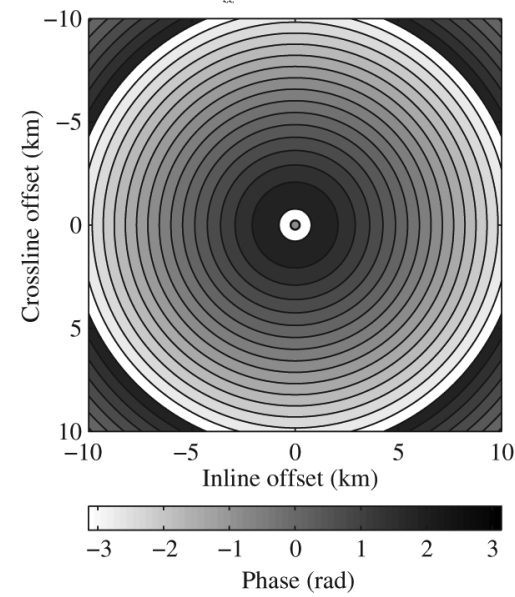

b)

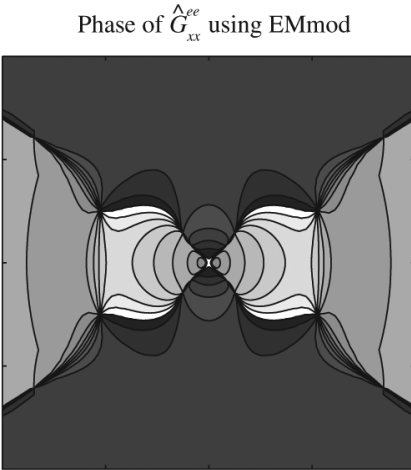

e)

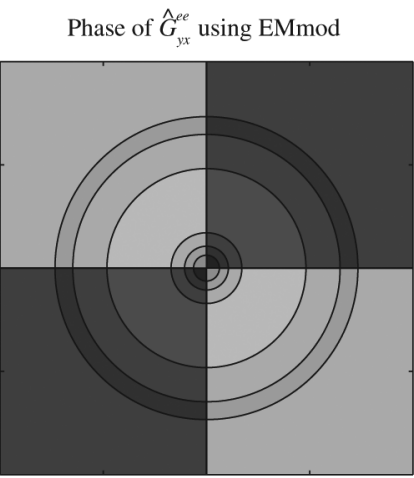

h)

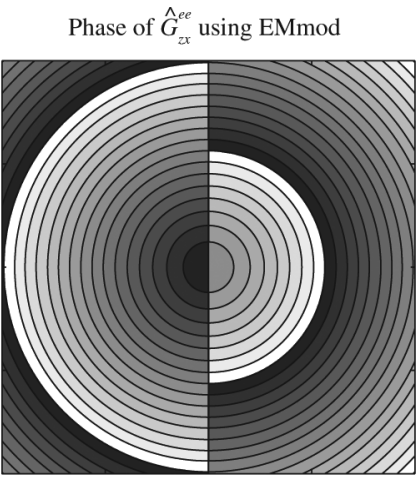

k)

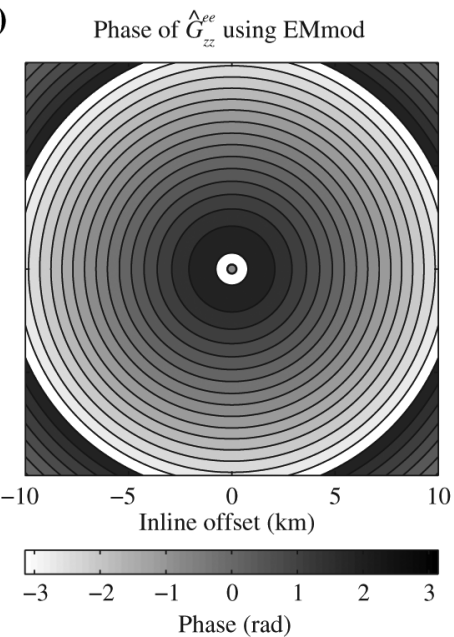

c)

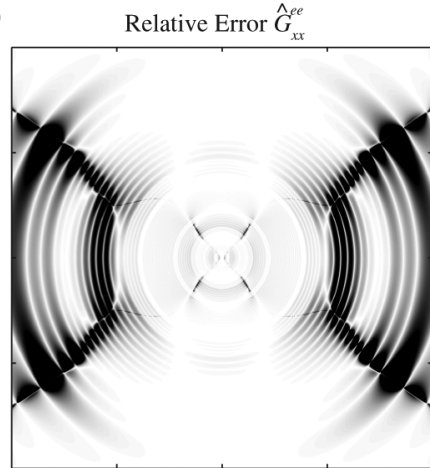

f)

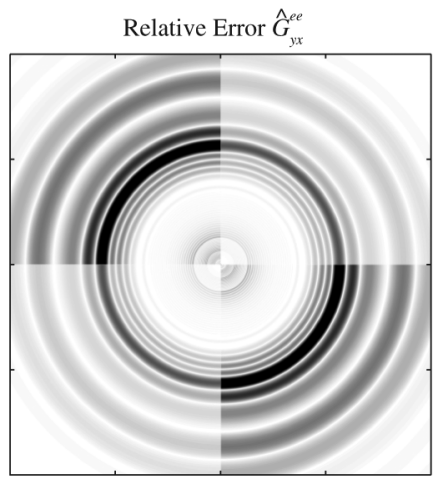

i)

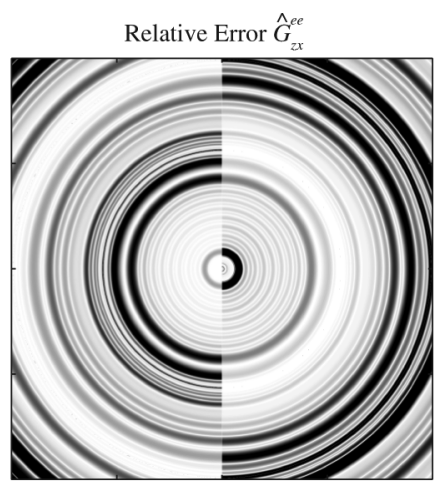

l)

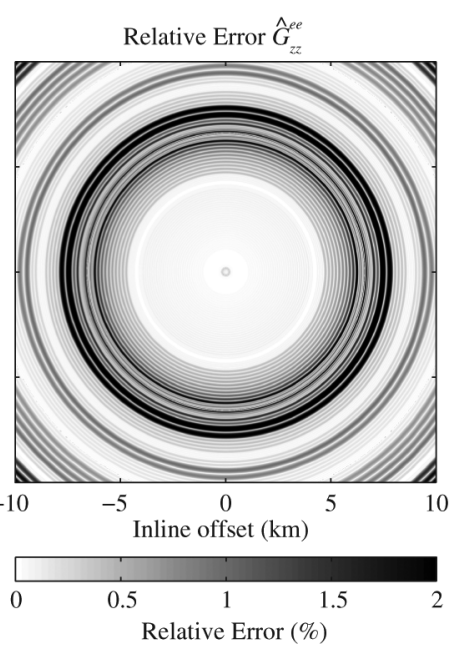

Figure 2. The same as Figure 1, but for the phase. Panels (a-c) show the phase of the half-space solution, the phase of the solution computed with EMmod and the relative error between the two for electric inline oriented receivers and an electric inline oriented source $\left(\hat{G}_{x x}^{e e}\right)$. Panels $(\mathrm{d}-\mathrm{f})$ show the same for $G_{y x}^{e e}$, panels $(\mathrm{g}-\mathrm{i})$ for $G_{z x}^{e e}$, and panels $(\mathrm{j}-1)$ for $G_{z z}^{e e}$. 


$$
\begin{aligned}
\hat{G}_{y y ; s}^{e m}\left(\mathbf{x}, \mathbf{x}^{\prime}, \omega\right)= & \hat{G}_{y y ; s}^{e m ; i}\left(\mathbf{x}-\mathbf{x}^{\prime}, \omega\right) \\
& -\frac{\sin (2 \phi)}{4 \pi} \int_{\kappa=0}^{\infty}\left(\tilde{g}_{h z ; s}^{t m}+\tilde{g}_{h h ; s}^{t e}\right) \mathbf{J}_{2}(\kappa r) \kappa \mathrm{d} \kappa,
\end{aligned}
$$

$$
\begin{aligned}
\hat{G}_{z y ; s}^{e m}\left(\mathbf{x}, \mathbf{x}^{\prime}, \omega\right)= & \hat{G}_{z y ; s}^{e m ; i}\left(\mathbf{x}-\mathbf{x}^{\prime}, \omega\right) \\
& +\frac{\eta_{s} \cos (\phi)}{8 \pi \eta_{s}^{(v)}} \int_{\kappa=0}^{\infty} \tilde{g}_{z z ; s}^{t m} \mathrm{~J}_{1}(\kappa r) \frac{\kappa^{2}}{\Gamma_{s}} \mathrm{~d} \kappa
\end{aligned}
$$

The electric field generated by the $z$-component of a point source of the magnetic current type is given by

$$
\begin{aligned}
\hat{G}_{x z ; s}^{e m}\left(\mathbf{x}, \mathbf{x}^{\prime}, \omega\right)= & \hat{G}_{x z ; s}^{e m ; i}\left(\mathbf{x}-\mathbf{x}^{\prime}, \omega\right) \\
& +\frac{\zeta_{s} \sin (\phi)}{8 \pi \zeta_{s}^{(v)}} \int_{\kappa=0}^{\infty} \tilde{g}_{z z ; s}^{t e} \mathrm{~J}_{1}(\kappa r) \frac{\kappa^{2}}{\bar{\Gamma}_{s}} \mathrm{~d} \kappa, \\
\hat{G}_{y z ; s}^{e m}\left(\mathbf{x}, \mathbf{x}^{\prime}, \omega\right)= & \hat{G}_{y z ; s}^{e m ; i}\left(\mathbf{x}-\mathbf{x}^{\prime}, \omega\right) \\
& -\frac{\zeta_{s} \cos (\phi)}{8 \pi \zeta_{s}^{(v)}} \int_{\kappa=0}^{\infty} \tilde{g}_{z z ; s}^{t e} \mathrm{~J}_{1}(\kappa r) \frac{\kappa^{2}}{\bar{\Gamma}_{s}} \mathrm{~d} \kappa, \\
& \hat{G}_{z z ; s}^{e m}\left(\mathbf{x}, \mathbf{x}^{\prime}, \omega\right)=0 .
\end{aligned}
$$

Using the duality principle, we have also found expressions for the components of the magnetic field generated by electric and magnetic dipole sources. If the receivers are not located in the source layer, the electromagnetic field can be computed with similar expressions but without the direct field terms.

The attached computer code evaluates these integrals with a 61point Gauss-Kronrod integration routine at one depth level. Note that the line integral is not angle dependent. Once the integral is evaluated for one line at one depth level, the electromagnetic field for a $2 \mathrm{D}$ grid at that depth level can be computed by simply multiplying the result with the factor preceding the integral for the angles $\phi$ corresponding to any grid points on the $2 \mathrm{D}$ grid. An alternative to solving the integral with a quadrature method would be to use digital filters as proposed, for example, by Kong (2007). However, that would limit the application of the code to problems in the diffusive limit. Because we aimed at producing a computer code that can be

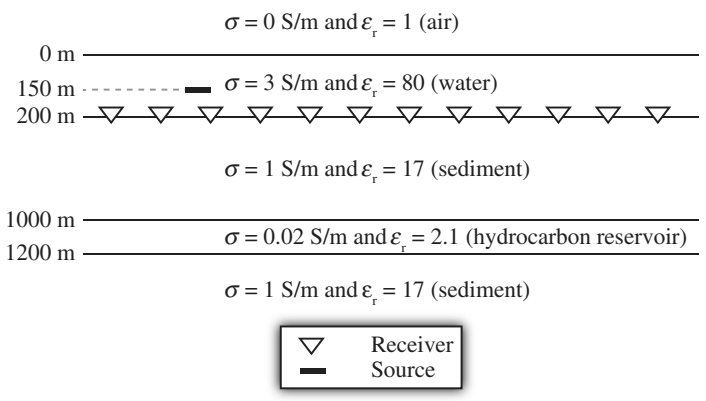

Figure 3. Model used for the comparison with the finite volume code. The layers of the medium are isotropic and characterized by conductivities $\sigma$ and relative electric permittivity $\varepsilon_{r}$. All layers have the magnetic permeability of a vacuum. applied to diffusion problems as well as to wave problems, we chose a quadrature method.

\section{EXAMPLES}

In this section, a set of examples is presented to illustrate the equations derived above and to show the capabilities of EMmod, the corresponding computer code. The first example compares the electric field computed by EMmod with an analytical solution in a medium of two homogeneous half-spaces. The analytical solution (Slob et al., 2010) neglects displacement currents, which is a valid approximation at low frequencies such as $0.5 \mathrm{~Hz}$ as used in this calculation. The upper half-space is nonconductive, whereas the lower half-space consists of a VTI medium with a vertical conductivity of $0.3 \mathrm{~S} / \mathrm{m}$ and a horizontal conductivity of $3 \mathrm{~S} / \mathrm{m}$. The relative electric permittivity is set to 80 , and the magnetic permeability is set to the value of free space. (Note that the effect of the relative electric permittivity at this frequency is so small, that it cannot be seen in the data. These values are given here because EMmod requires a value for all input parameters.) The source and the receivers are located 150 and $200 \mathrm{~m}$ below the interface between the two halfspaces, respectively. Thus, the vertical distance between the source and the receivers is $50 \mathrm{~m}$. The upper limit of the integral of the Hankel transformation is set to $k_{\max }=0.628625 \mathrm{~m}^{-1}$, which is twice the Nyquist wavenumber for a spatial sampling rate of $10 \mathrm{~m}$. All other parameter settings can be found in the file halfspacemod.scr included with the code. The electric field has been modeled for four different components in this setting: $\hat{G}_{x x}^{e e}, \hat{G}_{y x}^{e e}, \hat{G}_{z x}^{e e}$, and $\hat{G}_{z z}^{e e}$. Figure 1 shows for each of these components, the amplitude computed with the analytical half-space solution (Slob et al., 2010), the amplitude computed with EMmod, and the relative error between the two. It can be seen that the relative error is clearly less than $0.5 \%$ for all four situations. Slightly larger errors can appear at or near singularities as can be observed, for example, in the $x x$-component. Note that EMmod computes the Hankel integral only for a limited set of locations in the space domain and interpolates linearly or with pchip-interpolation to retrieve the fields at other locations. By increasing the number of Hankel integral evaluations, the precision can be increased significantly. Figure 2 shows the phase for the same components. For the phase, the relative error is mostly smaller than $0.5 \%$, except where the phase is close to zero. In that case, the computation of the relative error contains a division with a value that is almost zero, leading to higher relative error values.

The next example is a comparison of EMmod with a finite volume multigrid solver (Jönsthövel et al., 2006; Mulder, 2006, 2007). Using this solver allows us to compare the solution of EMmod for a more complicated model than just two homogeneous half-spaces. For this example, we have chosen a model representing a marine CSEM survey for hydrocarbon exploration (Figure 3). The source is a horizontal inline-oriented electric source emitting a signal as in the previous example at $0.5 \mathrm{~Hz}$ at a depth of $150 \mathrm{~m}$. The horizontal electric receivers are placed at the ocean bottom at a depth of $200 \mathrm{~m}$. The vertical electric receivers are at a depth of $175 \mathrm{~m}$. This latter choice was made because that is the location where the vertical component in the multigrid solver is computed. Thus, interpolating the vertical electric field across a layer interface, where it would not be continuous, is avoided. The electric fields $\hat{G}_{x x}^{e e}, \hat{G}_{y x}^{e e}$, and $\hat{G}_{z x}^{e e}$ computed with the multigrid solver and EMmod as well as the relative error between the two are plotted in Figure 4. The error is again mostly small (i.e., around $0.5 \%$ ), but there are areas of higher 
error toward the edges of the receiver array and close to the source. The multigrid solver forces the field to zero at the model boundary, which alters the solution. Furthermore, regularizing the stretched grid used in the multigrid solver introduces another source of inac- curacies. Discrepancies close to the source stem from the different size of the source. In the multigrid solver, the source has the size of the volume element where the source is located, and the source is a point source with no extension in any direction in EMmod. a)

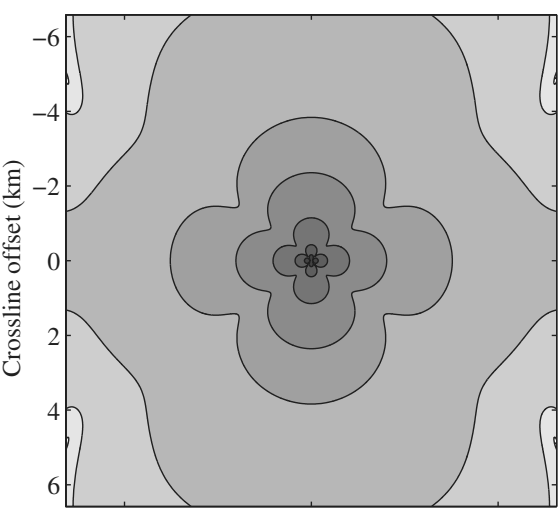

d)

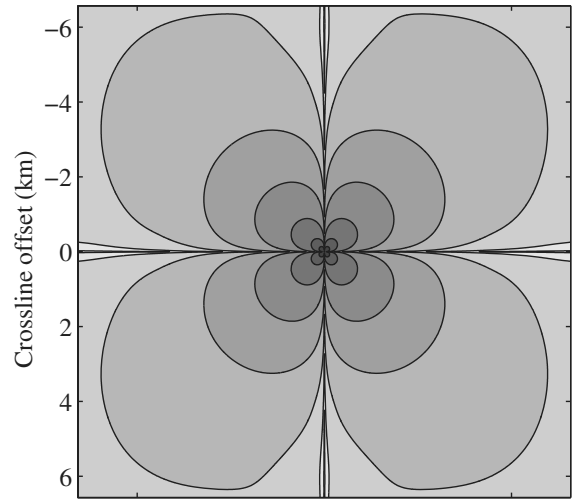

g)

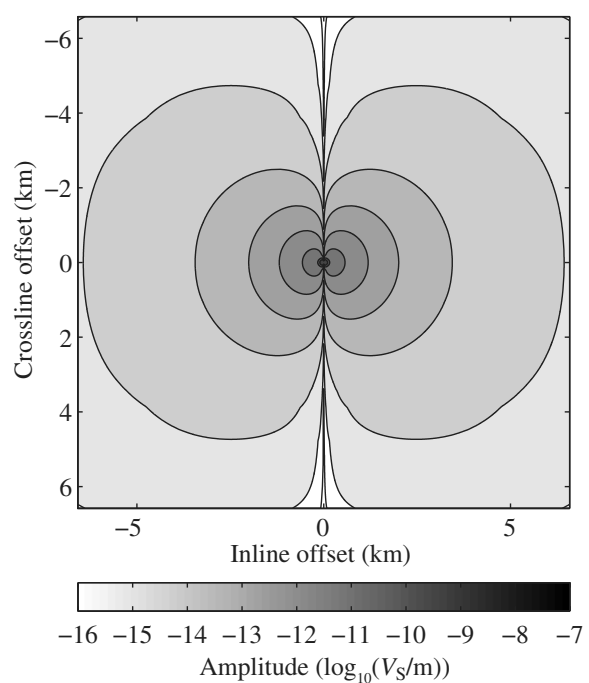

b) Amplitude of $\hat{G}_{x x}^{e e}$ using EMmod

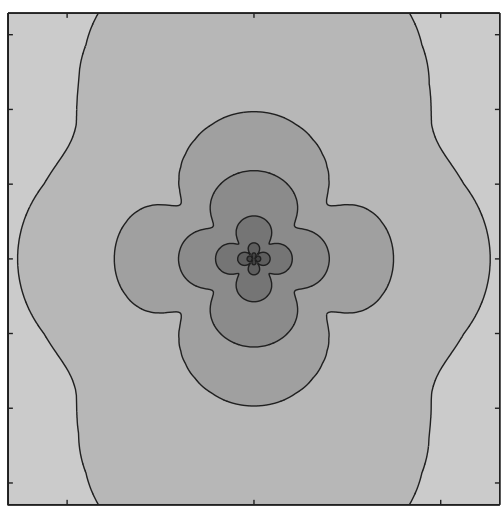

e) Amplitude of $\hat{G}_{v x}^{e e}$ using EMmod

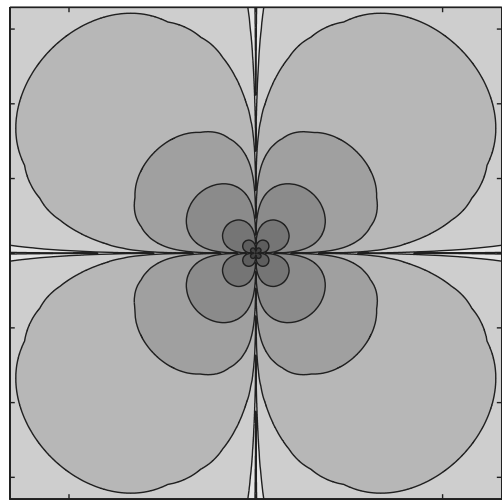

h) Amplitude of $\hat{G}_{z x}^{e e}$ using EMmod

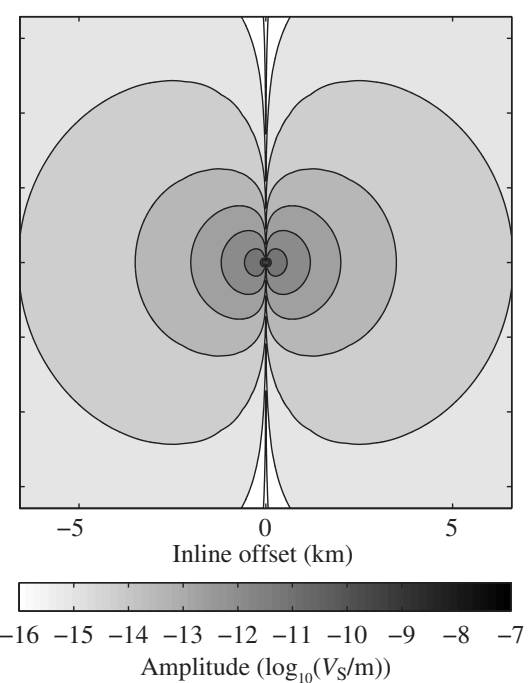

c)

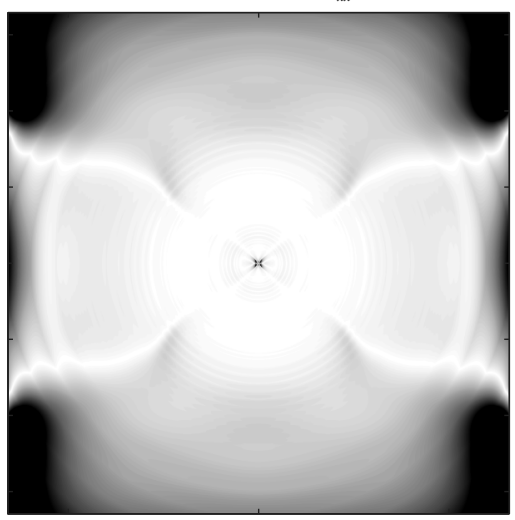

f)

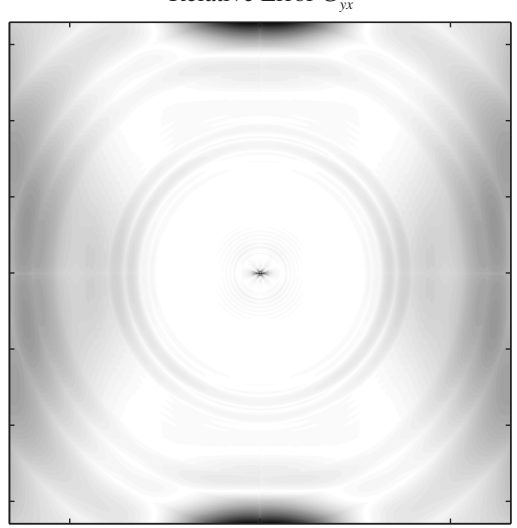

i)

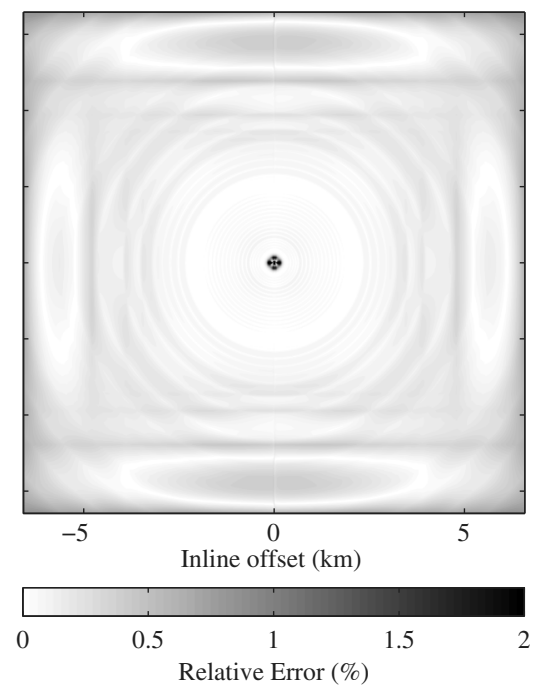

Figure 4. Comparison between the solution of the multigrid finite volume code and EMmod with respect to the amplitude. Panels (a-c) show the multigrid finite volume solution, the solution computed with EMmod and the relative error between the two for $G_{x x}^{e e}$. Panels (d-f) show the same for $\hat{G}_{y x}^{e e}$ and panels $(\mathrm{g}-\mathrm{i})$ for $\hat{G}_{z x}^{e e}$. 
Therefore, the small differences encountered between the two codes can be attributed to issues related to the multigrid solver.

The multigrid solver computes the electric field on a $3 \mathrm{D}$ grid, which allows us to use equation 6 to compute the magnetic fields. Those magnetic fields and the corresponding solution computed with EMmod as well as the relative error between the two are shown in Figure 5 for $\hat{G}_{x x}^{m e}, \hat{G}_{y x}^{m e}$, and $\hat{G}_{z x}^{m e}$. Again, the relative error is mostly around $0.5 \%$ except close to the source and toward the edges of the receiver array. Note that an additional source of error in the magnetic field of the multigrid solution stems from the decreased a) Amplitude of $\hat{G}^{\text {me }}$ using the multigrid code

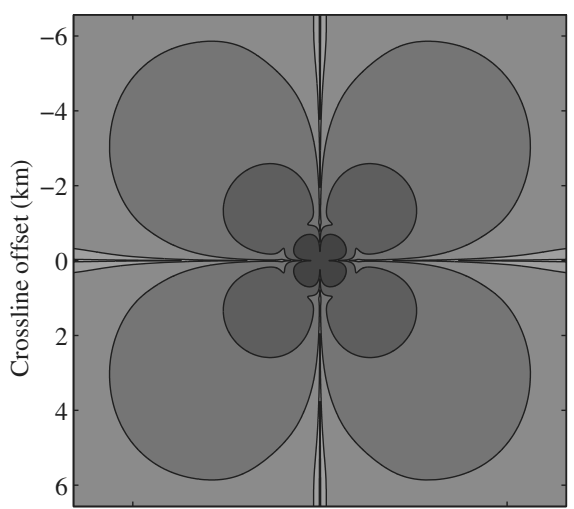

d)

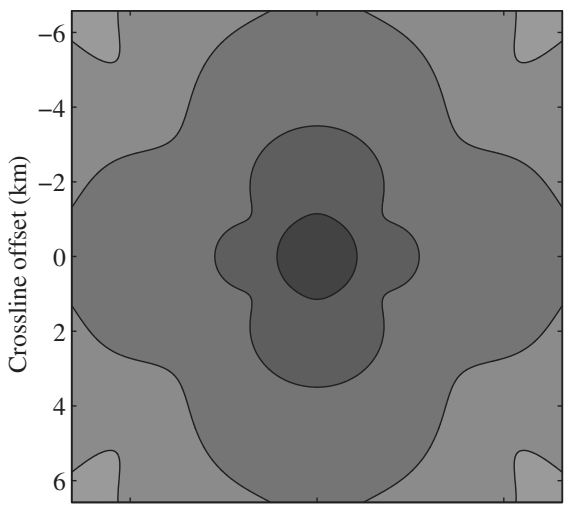

g) Amplitude of $\hat{G}_{z x}^{\mathrm{me}}$ using the multigrid code

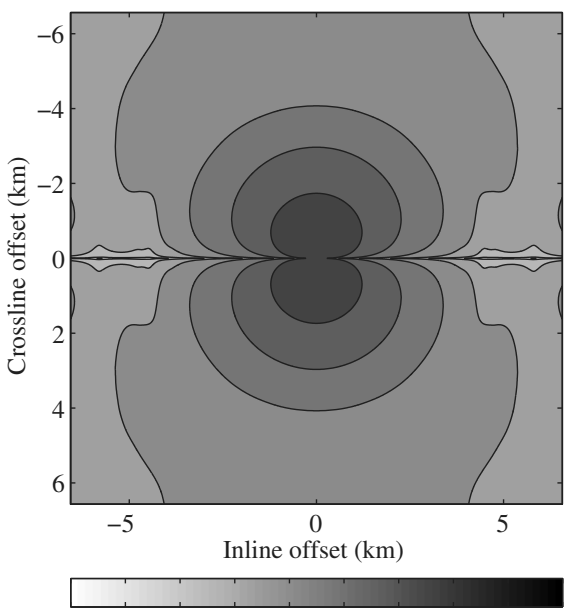

$\begin{array}{llllllllll}-16 & -15 & -14 & -13 & -12 & -11 & -10 & -9 & -8 & -7\end{array}$ Amplitude $\left(\log _{10}(\right.$ As $\left./ \mathrm{m})\right)$ b)

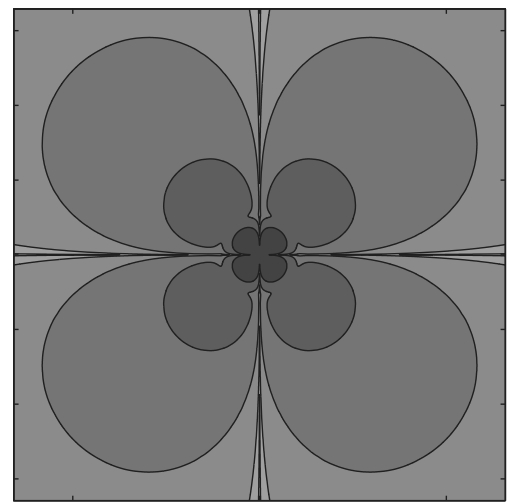

e)

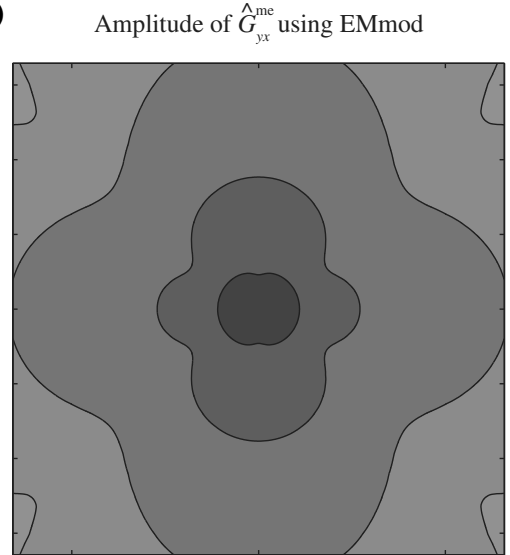

h)

Amplitude of $\hat{G}_{z x}^{\text {me }}$ using EMmod

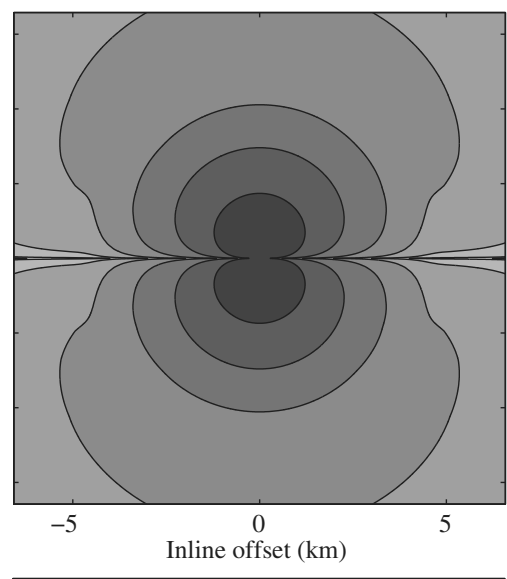

$\begin{array}{llllllllll}-16 & -15 & -14 & -13 & -12 & -11 & -10 & -9 & -8 & -7\end{array}$ Amplitude $\left(\log _{10}(\right.$ As $\left./ \mathrm{m})\right)$ c) Relative Error $\hat{G}_{x x}^{\text {me }}$

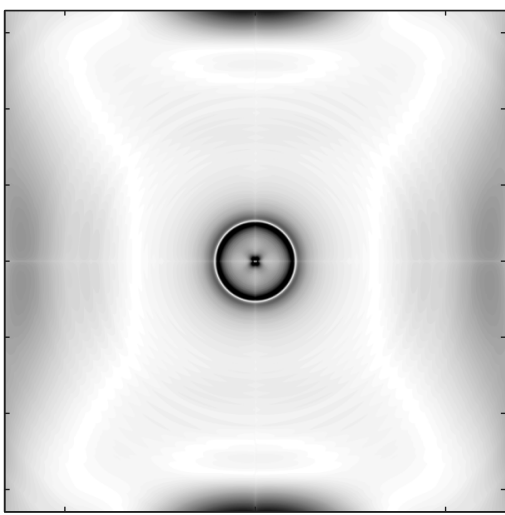

f) Relative Error $\hat{G}_{v x}^{\mathrm{me}}$

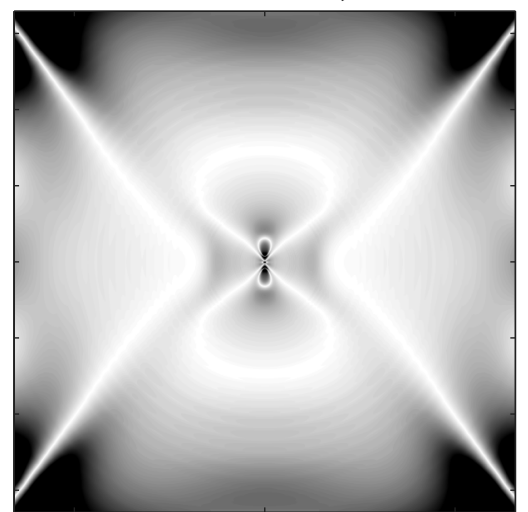

i)

Relative Error $\hat{G}_{z x}^{\mathrm{me}}$

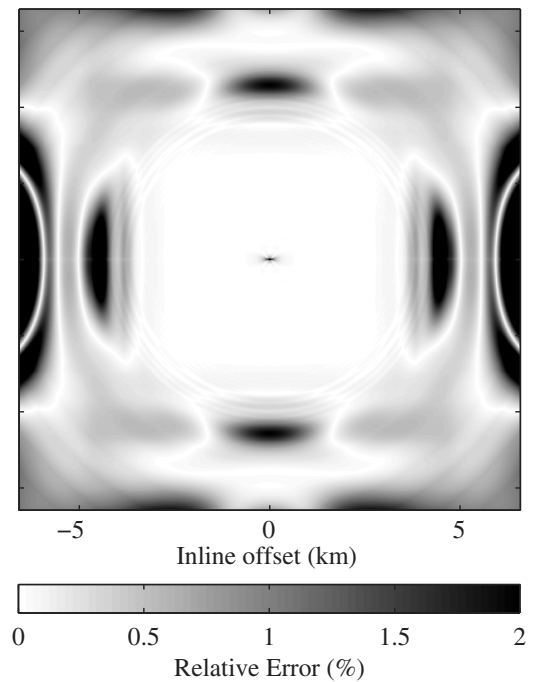

Figure 5. Same as Figure 4 but for magnetic field receivers. Panels (a-c) show $\hat{G}_{x x}^{m e}$, panels (d-f) show $\hat{G}_{y x}^{m e}$, and panels (g-i) show $\hat{G}_{z x}^{m e}$. 
precision due to using numerical derivatives in equation 6 . This possibly leads to the large circular error observed for $\hat{G}_{x x}^{m e}$ at approximately $1 \mathrm{~km}$ offset.

The third example is a GPR application. Because GPR is a highfrequency technology, the electromagnetic fields behave like waves. The model consists of a layer sandwiched between two homogeneous half-spaces. The conductivity and the relative electric permittivity of each material as well as the source and receiver geometry are given in the left side of Figure 6. Note that unlike in traditional GPR, the receivers are located $0.5 \mathrm{~m}$ below the source. The reason for this choice lies in the nature of EMmod. If the source and the receivers were both located at the surface with no vertical distance between them, the specular reflection from the surface would have an infinite bandwidth in the wavenumber domain, which is difficult to cope with using a numerical integration scheme for the Hankel transformation. The direct field does not pose a problem because it is computed in the space domain directly. The vertical distance between the source and receivers can be minimized by increasing the bandwidth of the integral. A rule of thumb for how to choose the upper bound of the integral for a given vertical distance $h$ between the source and receivers is given by the following relation (similar to the expression used in Lambot et al., 2007):

$$
k_{\max }=\sqrt{\left(\frac{10}{h}\right)^{2}+\left(\frac{\omega}{c}\right)^{2}}
$$

with $\omega$ being the angular frequency and $c$ the speed of light. The factor 10 ensures that the signal has decayed to a magnitude of $e^{-10}$ at the resulting $k_{\max }$. We have chosen an upper bound for the integral of $k_{\max }=160 \mathrm{~m}^{-1}$, which is approximately 3.5 times larger than indicated by equation 129 in order to be sure to avoid artifacts. Tapering the response in the wavenumber domain before the integral is carried out may also help stabilize the solution. The resulting data computed with EMmod for $\hat{G}_{x x}^{e e}$ are shown on the right side of Figure 6 The data are convolved with a Ricker wavelet with a central frequency of $250 \mathrm{MHz}$, and a gain function $\left(1+\left|t^{3}\right|\right.$ with $t$ being the time in nanoseconds) is applied. The theoretical arrival times of the direct wave (red), the wave that is refracted at the surface (yellow), and the wave that is reflected at the subsurface interface (green) are indicated with solid lines. In the left side of the same figure, arrows correspondingly colored show the travel path associated with these events. The theoretical traveltimes cor- respond very well with the events in the data, confirming that EMmod also works in the high-frequency regime. All the other visible events in the data are multiple reverberations of either the refracted or the reflected wave as they typically occur in GPR. The signal that is reflected at the surface coincides with the direct wave due to the proximity of the source to the surface.

\section{CONCLUSIONS}

Starting with the Maxwell equations, we have shown in a VTIlayered earth that if the vertical electric (TM-mode) and vertical magnetic (TE-mode) field components are known, the whole electromagnetic field can be derived from those two. Moreover, through the duality principle, the vertical magnetic field is known if the vertical electric field is known. Thus, by determining the vertical electric field, we are able to derive the full 3D electromagnetic response in a 1D medium consisting of VTI layers.

Our solution is accompanied by the computer code EMmod written in $\mathrm{C}$ and Fortran, which uses our findings to model the 3D electromagnetic field in a $1 \mathrm{D}$ earth for diffusive methods such as CSEM as well as for wave methods such as GPR. The user can freely choose between electric or magnetic dipole sources and receivers and place them anywhere in the medium. The capabilities and the correctness of the code are illustrated with three examples, which show (1) the diffusive electric field in two homogeneous halfspaces of which the lower one consists of a VTI medium, (2) the diffusive electromagnetic field in an isotropic but otherwise more complex medium, and (3) the response of an electromagnetic wave in the high-frequency limit in an isotropic layer sandwiched by two homogeneous isotropic half-spaces.

\section{ACKNOWLEDGMENTS}

This work is supported by the Netherlands Research Center for Integrated Solid Earth Science and the Dutch Technology Foundation STW (grant DCB.7913). We would like to thank C. Schoemaker for commenting on an early version of the manuscript and A. Schaller and J. Brackenhoff for testing the latest version of EMmod. We also thank W. Mulder for giving us access to the multigrid code used in the second example. Finally, we thank three anonymous reviewers for their comments.

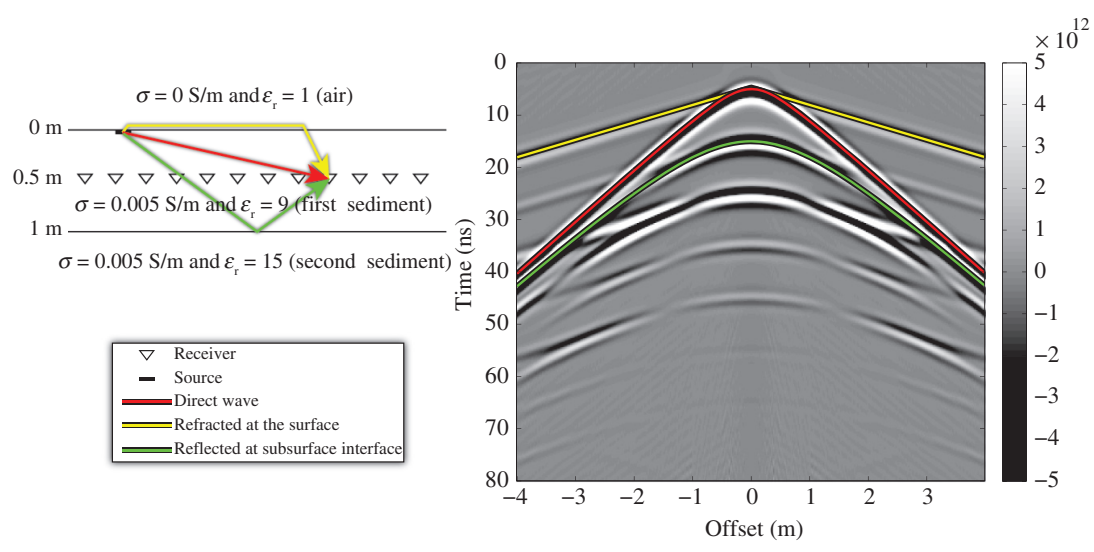

Figure 6. GPR example: The left side shows the model used, and the right side shows the computed data. The theoretical traveltimes of three events are superimposed with colored lines. 


\section{THE VERTICAL COMPONENT OF THE MAGNETIC FIELD}

The vertical component of the magnetic field is obtained from equations 75-104 as

$$
\begin{gathered}
\tilde{G}_{z x ; s}^{m m}=\tilde{G}_{z x ; s}^{m m ; i}+\frac{\mathrm{i} k_{x}}{2 \zeta_{s}^{(v)}}\left\{\bar{P}_{s}^{u-} \bar{W}_{s}^{u}-\bar{P}_{s}^{d-} \bar{W}_{s}^{d}\right\}, \\
\tilde{G}_{z y ; s}^{m m}=\tilde{G}_{z y ; s}^{m m ; i}+\frac{\mathrm{i} k_{y}}{2 \zeta_{s}^{(v)}}\left\{\bar{P}_{s}^{u-} \bar{W}_{s}^{u}-\bar{P}_{s}^{d-} \bar{W}_{s}^{d}\right\}, \\
\tilde{G}_{z z ; s}^{m m}=\tilde{G}_{z z ; s}^{m m ; i}+\frac{\zeta_{s} \kappa^{2}}{2\left(\zeta_{s}^{(v)}\right)^{2} \bar{\Gamma}_{s}}\left\{\bar{P}_{s}^{u+} \bar{W}_{s}^{u}+\bar{P}_{s}^{d+} \bar{W}_{s}^{d}\right\},
\end{gathered}
$$

where the vertical wavenumber for the TE mode is given by

$$
\bar{\Gamma}=\sqrt{\zeta / \zeta^{(v)}} \sqrt{\kappa^{2}+\bar{\gamma}^{2}}
$$

The incident fields and the up- and downgoing fields are written as

$$
\begin{gathered}
\tilde{G}_{z x ; s}^{m m ; i}=-\frac{\mathrm{i} k_{x} \partial_{z}}{\zeta_{s}^{(v)}} \frac{\exp \left(-\bar{\Gamma}_{s}\left|z-z^{\prime}\right|\right)}{2 \bar{\Gamma}_{s}} \\
\tilde{G}_{z y ; s}^{m m ; i}=-\frac{\mathrm{i} k_{y} \partial_{z}}{\zeta_{s}^{(v)}} \frac{\exp \left(-\bar{\Gamma}_{s}\left|z-z^{\prime}\right|\right)}{2 \bar{\Gamma}_{s}} \\
\tilde{G}_{z z ; s}^{m m ; i}=\frac{\partial_{z} \partial_{z}-\eta_{s} \zeta_{s}}{\zeta_{s}^{(v)}} \frac{\exp \left(-\bar{\Gamma}_{s}\left|z-z^{\prime}\right|\right)}{2 \bar{\Gamma}_{s}} \\
\bar{P}_{s}^{u \pm}=\frac{\bar{R}_{s}^{+}}{\bar{M}_{s}}\left(\exp \left(-\bar{\Gamma}_{s} d^{+}\right) \pm \bar{R}_{s}^{-} \exp \left[-\bar{\Gamma}_{s}\left(d_{s}+d^{-}\right)\right]\right) \\
\bar{P}_{s}^{d \pm}=\frac{\bar{R}_{s}^{-}}{\bar{M}_{s}}\left(\exp \left(-\bar{\Gamma}_{s} d^{-}\right) \pm \bar{R}_{s}^{+} \exp \left[-\bar{\Gamma}_{s}\left(d_{s}+d^{+}\right)\right]\right)
\end{gathered}
$$

In these equations, the factor $\bar{M}_{s}$ and the TE-mode global reflection coefficients are given by

$$
\begin{array}{r}
\bar{M}_{s}=1-\bar{R}_{s}^{-} \bar{R}_{s}^{+} \exp \left(-2 \bar{\Gamma}_{s} d_{s}\right), \\
\bar{R}_{n}^{ \pm}=\frac{\bar{r}_{n}^{ \pm}+\bar{R}_{n \pm 1}^{ \pm} \exp \left(-2 \bar{\Gamma}_{n \pm 1} d_{n \pm 1}\right)}{1+\bar{r}_{n}^{ \pm} \bar{R}_{n \pm 1}^{ \pm} \exp \left(-2 \bar{\Gamma}_{n \pm 1} d_{n \pm 1}\right)},
\end{array}
$$

with local reflection coefficients

$$
\bar{r}_{n}^{ \pm}=\frac{\zeta_{n \pm 1} \bar{\Gamma}_{n}-\zeta_{n} \bar{\Gamma}_{n \pm 1}}{\zeta_{n \pm 1} \bar{\Gamma}_{n}+\zeta_{n} \bar{\Gamma}_{n \pm 1}}
$$

The vertical magnetic field generated by electric point sources are obtained as

$$
\begin{gathered}
\tilde{G}_{z x ; s}^{m e}=\tilde{G}_{z x ; s}^{m e ; i}+\frac{\zeta_{s} \mathrm{i} k_{y}}{2 \zeta_{s}^{(v)} \bar{\Gamma}_{s}}\left(\bar{P}_{s}^{u+} \bar{W}_{s}^{u}+\bar{P}_{s}^{d+} \bar{W}_{s}^{d}\right), \\
\tilde{G}_{z y ; s}^{m e}=\tilde{G}_{z y ; s}^{m e ; i}-\frac{\zeta_{s} \mathrm{i} k_{x}}{2 \zeta_{s}^{(v)} \bar{\Gamma}_{s}}\left(\bar{P}_{s}^{u+} \bar{W}_{s}^{u}+\bar{P}_{s}^{d+} \bar{W}_{s}^{d}\right), \\
\tilde{G}_{z z ; s}^{m e}=0 .
\end{gathered}
$$

The incident fields are given by

$$
\begin{array}{r}
\tilde{G}_{z x ; s}^{m e ; i}=\frac{\zeta_{s} \mathrm{i} k_{y}}{\zeta_{s}^{(v)}} \frac{\exp \left(-\bar{\Gamma}_{s}\left|z-z^{\prime}\right|\right)}{2 \bar{\Gamma}_{s}}, \\
\tilde{G}_{z y ; s}^{m e ; i}=-\frac{\zeta_{s} \mathrm{i} k_{x}}{\zeta_{s}^{(v)}} \frac{\exp \left(-\bar{\Gamma}_{s}\left|z-z^{\prime}\right|\right)}{2 \bar{\Gamma}_{s}} .
\end{array}
$$

In the layers above the source, we find

$$
\begin{aligned}
& \tilde{G}_{z x ; n}^{m m}=\frac{\mathrm{i} k_{x}}{2 \zeta_{n}^{(v)}} \bar{P}_{n}^{u-}\left(\bar{W}_{n}^{u}+\bar{R}_{n}^{-} \exp \left(-\bar{\Gamma}_{n} d_{n}\right) \bar{W}_{n}^{d}\right), \\
& \tilde{G}_{z y ; n}^{m m}=\frac{\mathrm{i} k_{y}}{2 \zeta_{n}^{(v)}} \bar{P}_{n}^{u-}\left(\bar{W}_{n}^{u}+\bar{R}_{n}^{-} \exp \left(-\bar{\Gamma}_{n} d_{n}\right) \bar{W}_{n}^{d}\right), \\
& \tilde{G}_{z z ; n}^{m m}=\frac{\zeta_{s} \kappa^{2}}{2 \zeta_{s}^{(v)} \zeta_{n}^{(v)} \bar{\Gamma}_{s}} \bar{P}_{n}^{u+}\left(\bar{W}_{n}^{u}+\bar{R}_{n}^{-} \exp \left(-\bar{\Gamma}_{n} d_{n}\right) \bar{W}_{n}^{d}\right),
\end{aligned}
$$

and

$$
\begin{gathered}
\tilde{G}_{z x ; n}^{m e}=\frac{\zeta_{s} \mathrm{i} k_{y}}{2 \zeta_{n}^{(v)} \bar{\Gamma}_{s}} \bar{P}_{n}^{u+}\left(\bar{W}_{n}^{u}+\bar{R}_{n}^{-} \exp \left(-\bar{\Gamma}_{n} d_{n}\right) \bar{W}_{n}^{d}\right), \\
\tilde{G}_{z y ; n}^{m e}=-\frac{\zeta_{s} \mathrm{i} k_{x}}{2 \zeta_{n}^{(v)} \bar{\Gamma}_{s}} \bar{P}_{n}^{u+}\left(\bar{W}_{n}^{u}+\bar{R}_{n}^{-} \exp \left(-\bar{\Gamma}_{n} d_{n}\right) \bar{W}_{n}^{d}\right), \\
\tilde{G}_{z z ; n}^{m e}=0
\end{gathered}
$$

for $n=s-1$ and $0 \leq n<s-1$, and where the factors $\bar{P}_{n}^{ \pm}$are given by

$$
\bar{P}_{s-1}^{u \pm}=\frac{\left(1+\bar{R}_{s}^{-}\right)\left\{ \pm \exp \left(-\bar{\Gamma}_{s} d^{-}\right)+\bar{R}_{s}^{+} \exp \left[-\bar{\Gamma}_{s}\left(d_{s}+d^{+}\right)\right]\right\}}{\bar{M}_{s}\left[1+\bar{R}_{s-1}^{-} \exp \left(-2 \bar{\Gamma}_{s-1} d_{s-1}\right)\right]},
$$

$$
\bar{P}_{n}^{u \pm}=\bar{P}_{s-1}^{u \pm} \prod_{m=n}^{s-2} \frac{\left(1+\bar{R}_{m+1}^{-}\right) \exp \left(-\bar{\Gamma}_{m+1} d_{m+1}\right)}{1+\bar{R}_{m}^{-} \exp \left(-2 \bar{\Gamma}_{m} d_{m}\right)}
$$


In the layers below the source, we find

$$
\begin{aligned}
& \tilde{G}_{z x ; n}^{m m}=\frac{\mathrm{i} k_{x}}{2 \zeta_{n}^{(v)}} \bar{P}_{n}^{d-}\left(\bar{W}_{n}^{d}+\bar{R}_{n}^{+} \exp \left(-\bar{\Gamma}_{n} d_{n}\right) \bar{W}_{n}^{u}\right), \\
& \tilde{G}_{z y ; n}^{m m}=\frac{\mathrm{i} k_{y}}{2 \zeta_{n}^{(v)}} \bar{P}_{n}^{d-}\left(\bar{W}_{n}^{d}+\bar{R}_{n}^{+} \exp \left(-\bar{\Gamma}_{n} d_{n}\right) \bar{W}_{n}^{u}\right), \\
& \tilde{G}_{z z ; n}^{m m}=\frac{\zeta_{s} \kappa^{2}}{2 \zeta_{s}^{(v)} \zeta_{n}^{(v)} \bar{\Gamma}_{s}} \bar{P}_{n}^{d+}\left(\bar{W}_{n}^{d}+\bar{R}_{n}^{-} \exp \left(-\bar{\Gamma}_{n} d_{n}\right) \bar{W}_{n}^{u}\right),
\end{aligned}
$$

and

$$
\begin{gathered}
\tilde{G}_{z x ; n}^{m e}=\frac{\zeta_{s} \mathrm{i} k_{y}}{2 \zeta_{n}^{(v)} \bar{\Gamma}_{s}} \bar{P}_{n}^{d+}\left(\bar{W}_{n}^{d}+\bar{R}_{n}^{+} \exp \left(-\bar{\Gamma}_{n} d_{n}\right) \bar{W}_{n}^{u}\right), \\
\tilde{G}_{z y ; n}^{m e}=-\frac{\zeta_{s} \mathrm{i} k_{x}}{2 \zeta_{n}^{(v)} \bar{\Gamma}_{s}} \bar{P}_{n}^{d+}\left(\bar{W}_{n}^{d}+\bar{R}_{n}^{+} \exp \left(-\bar{\Gamma}_{n} d_{n}\right) \bar{W}_{n}^{u}\right), \\
\tilde{G}_{z z ; n}^{m e}=0,
\end{gathered}
$$

for $n=s+1$ and $s+1<n \leq N+1$ and the factors $\bar{P}_{n}^{ \pm}$are given by

$$
\begin{gathered}
\bar{P}_{s+1}^{d \pm}=\frac{\left(1+\bar{R}_{s}^{+}\right)\left\{\exp \left(-\bar{\Gamma}_{s} d^{+}\right) \pm \bar{R}_{s}^{-} \exp \left[-\bar{\Gamma}_{s}\left(d_{s}+d^{-}\right)\right]\right\}}{\bar{M}_{s}\left[1+\bar{R}_{s+1}^{+} \exp \left(-2 \bar{\Gamma}_{s+1} d_{s+1}\right)\right]}, \\
\bar{P}_{n}^{d \pm}=\bar{P}_{s+1}^{d \pm} \prod_{m=s+2}^{n} \frac{\left(1+\bar{R}_{m-1}^{+}\right) \exp \left(-\bar{\Gamma}_{m-1} d_{m-1}\right)}{1+\bar{R}_{m}^{+} \exp \left(-2 \bar{\Gamma}_{m} d_{m}\right)} .
\end{gathered}
$$

With these expressions, the horizontal components of the electric and magnetic fields can be constructed.

\section{APPENDIX B}

\section{THE HORIZONTAL ELECTRIC FIELD COMPONENTS}

The horizontal electric field generated by horizontal electric current point sources and received in the source layer are obtained as

$$
\begin{aligned}
\tilde{G}_{x x ; s}^{e e}= & \tilde{G}_{x x ; s}^{e e ; i}-\frac{\left(\mathrm{i} k_{x}\right)^{2} \Gamma_{s}}{2 \eta_{s} \kappa^{2}}\left(P_{s}^{u-} W_{s}^{u}+P_{s}^{d-} W_{s}^{d}\right) \\
+ & \frac{\zeta_{s}\left(\mathrm{i} k_{y}\right)^{2}}{2 \kappa^{2} \bar{\Gamma}_{s}}\left(\bar{P}_{s}^{u+} \bar{W}_{s}^{u}+\bar{P}_{s}^{d+} \bar{W}_{s}^{d}\right), \\
\tilde{G}_{x y ; s}^{e e}= & \tilde{G}_{x y, s}^{e e ; i}-\frac{\mathrm{i} k_{x} \mathrm{i} k_{y} \Gamma_{s}}{2 \eta_{s} \kappa^{2}}\left(P_{s}^{u-} W_{s}^{u}+P_{s}^{d-} W_{s}^{d}\right) \\
& -\frac{\zeta_{s} \mathrm{i} k_{x} \mathrm{i} k_{y}}{2 \kappa^{2} \bar{\Gamma}_{s}}\left(\bar{P}_{s}^{u+} \bar{W}_{s}^{u}+\bar{P}_{s}^{d+} \bar{W}_{s}^{d}\right),
\end{aligned}
$$

$$
\tilde{G}_{x z ; s}^{e e}=\tilde{G}_{x z ; s}^{e e ; i}-\frac{\mathrm{i} k_{x}}{2 \eta_{s}^{(v)}}\left(P_{s}^{u+} W_{s}^{u}-P_{s}^{d+} W_{s}^{d}\right) .
$$

The function $\tilde{G}_{y x ; n}^{e e}=\tilde{G}_{x y ; n}^{e e}$ for any layer $n, \tilde{G}_{y y ; n}^{e e}$ is equal to $\tilde{G}_{x x ; n}^{e e}$, and $\tilde{G}_{y z ; n}^{e e}$ is equal to $\tilde{G}_{x z ; n}^{e e}$, for any $n$ when $k_{x}$ and $k_{y}$ are interchanged. The horizontal electric field components generated by vertical electric dipoles can also be obtained from the vertical electric field generated by horizontal electric dipoles through reciprocity, by taking $k_{x}=-k_{x}, k_{y}=-k_{y}$ and interchanging $z$ and $z^{\prime}$. This will lead to the same result. The incident fields are given by

$$
\begin{aligned}
\tilde{G}_{x x ; s}^{e e j i}= & \frac{\left(\mathrm{i} k_{x}\right)^{2} \Gamma_{s}}{2 \eta_{s} \kappa^{2}} \exp \left(-\Gamma_{s}\left|z-z^{\prime}\right|\right) \\
& +\frac{\zeta_{s}\left(\mathrm{i} k_{y}\right)^{2}}{2 \bar{\Gamma}_{s} \kappa^{2}} \exp \left(-\bar{\Gamma}_{s}\left|z-z^{\prime}\right|\right), \\
\tilde{G}_{x y ; s}^{e e j i}= & \frac{\mathrm{i} k_{x} \mathrm{i} k_{y} \Gamma_{s}}{2 \eta_{s} \kappa^{2}} \exp \left(-\Gamma_{s}\left|z-z^{\prime}\right|\right) \\
& -\frac{\zeta_{s} \mathrm{i} k_{x} \mathrm{i} k_{y}}{2 \bar{\Gamma}_{s} \kappa^{2}} \exp \left(-\bar{\Gamma}_{s}\left|z-z^{\prime}\right|\right), \\
\tilde{G}_{x z ; s}^{e x ; i}= & -\frac{\mathrm{i} k_{x} \partial_{z}}{2 \Gamma_{s} \eta_{s}^{(v)}} \exp \left(-\Gamma_{s}\left|z-z^{\prime}\right|\right) .
\end{aligned}
$$

The horizontal electric field in the source layer and generated by magnetic dipoles is given by

$$
\begin{gathered}
\tilde{G}_{x x ; s}^{e m}=\tilde{G}_{x x ; s}^{e m ; i} \\
+\frac{\mathrm{i} k_{x} \mathrm{i} k_{y}}{2 \kappa^{2}}\left(P_{s}^{u+} W_{s}^{u}-P_{s}^{d+} W_{s}^{d}+\bar{P}_{s}^{u-} \bar{W}_{s}^{u}-\bar{P}_{s}^{d-} \bar{W}_{s}^{d}\right), \\
\tilde{G}_{x y ; s}^{e m}=\tilde{G}_{x y ; s}^{e m ; i}-\frac{\left(\mathrm{i} k_{x}\right)^{2}}{2 \kappa^{2}}\left(P_{s}^{u+} W_{s}^{u}-P_{s}^{d+} W_{s}^{d}\right) \\
+\frac{\left(\mathrm{i} k_{y}\right)^{2}}{2 \kappa^{2}}\left(\bar{P}_{s}^{u-} \bar{W}_{s}^{u}-\bar{P}_{s}^{d-} \bar{W}_{s}^{d}\right), \\
\tilde{G}_{x z ; s}^{e m}=\tilde{G}_{x z ; s}^{e m ; i}+\frac{\zeta_{s}}{\zeta_{s}^{(v)}} \frac{\mathrm{i} k_{y}}{2 \bar{\Gamma}_{s}}\left(\bar{P}_{s}^{u+} \bar{W}_{s}^{u}+\bar{P}_{s}^{d+} \bar{W}_{s}^{d}\right) .
\end{gathered}
$$

For any layer $n$, the function $\tilde{G}_{y x ; n}^{e m}$ is equal to $-\tilde{G}_{x y ; n}^{e m}$ and $\tilde{G}_{y z ; n}^{e m}$ is equal to $-\tilde{G}_{x z ; n}^{e m}$ when $k_{x}$ and $k_{y}$ are interchanged, and $\tilde{G}_{y y, n}^{e m}=$ $-\tilde{G}_{x x ; n}^{e m}$. The incident fields are given by

$$
\begin{aligned}
\tilde{G}_{x x ; s}^{e m ; i}= & \frac{\mathrm{i} k_{x} \mathrm{i} k_{y} \partial_{z}}{2 \kappa^{2} \Gamma_{s}} \exp \left(-\Gamma_{s}\left|z-z^{S}\right|\right) \\
& -\frac{\mathrm{i} k_{x} \mathrm{i} k_{y} \partial_{z}}{2 \kappa^{2} \bar{\Gamma}_{s}} \exp \left(-\bar{\Gamma}_{s}\left|z-z^{S}\right|\right), \\
\tilde{G}_{x y ; s}^{e m ; i}= & -\frac{\left(\mathrm{i} k_{x}\right)^{2} \partial_{z}}{2 \kappa^{2} \Gamma_{s}} \exp \left(-\Gamma_{s}\left|z-z^{S}\right|\right) \\
& -\frac{\left(\mathrm{i} k_{y}\right)^{2} \partial_{z}}{2 \kappa^{2} \bar{\Gamma}_{s}} \exp \left(-\bar{\Gamma}_{s}\left|z-z^{S}\right|\right),
\end{aligned}
$$




$$
\tilde{G}_{x z ; s}^{e m ; i}=\frac{\zeta_{s} \mathrm{i} k_{y}}{2 \zeta_{s}^{(v)} \bar{\Gamma}_{s}} \exp \left(-\bar{\Gamma}_{s}\left|z-z^{S}\right|\right) .
$$

In the layers above the source, we find

$$
\begin{aligned}
\tilde{G}_{x x ; n}^{e e}= & -\frac{\left(\mathrm{i} k_{x}\right)^{2} \Gamma_{n}}{2 \eta_{n} \kappa^{2}} P_{n}^{u-}\left(W_{n}^{u}-R_{n}^{-} \exp \left(-\Gamma_{n} d_{n}\right) W_{n}^{d}\right) \\
& +\frac{\zeta_{s}\left(\mathrm{i} k_{y}\right)^{2}}{2 \kappa^{2} \bar{\Gamma}_{s}} \bar{P}_{n}^{u+}\left(\bar{W}_{n}^{u}+\bar{R}_{n}^{-} \exp \left(-\bar{\Gamma}_{n} d_{n}\right) \bar{W}_{n}^{d}\right), \\
\tilde{G}_{x y ; s}^{e e}=- & -\frac{\mathrm{i} k_{x} \mathrm{i} k_{y} \Gamma_{n}}{2 \eta_{n} \kappa^{2}} P_{n}^{u-}\left(W_{n}^{u}-R_{n}^{-} \exp \left(-\Gamma_{n} d_{n}\right) W_{n}^{d}\right) \\
& -\frac{\zeta_{s} \mathrm{i} k_{x} \mathrm{i} k_{y}}{2 \kappa^{2} \bar{\Gamma}_{s}} \bar{P}_{n}^{u+}\left(\bar{W}_{n}^{u}+\bar{R}_{n}^{-} \exp \left(-\bar{\Gamma}_{n} d_{n}\right) \bar{W}_{n}^{d}\right), \\
\tilde{G}_{x z ; s}^{e e}= & -\frac{\eta_{s} \mathrm{i} k_{x} \Gamma_{n}}{2 \eta_{n} \eta_{s}^{(v)} \Gamma_{s}} P_{n}^{u+}\left(W_{n}^{u}-R_{n}^{-} \exp \left(-\Gamma_{n} d_{n}\right) W_{n}^{d}\right) .
\end{aligned}
$$

In the layers below the source, we find

$$
\begin{aligned}
\tilde{G}_{x x ; n}^{e e}= & \frac{\left(\mathrm{i} k_{x}\right)^{2} \Gamma_{n}}{2 \eta_{n} \kappa^{2}} P_{n}^{d-}\left(W_{n}^{d}-R_{n}^{+} \exp \left(-\Gamma_{n} d_{n}\right) W_{n}^{u}\right) \\
& +\frac{\zeta_{s}\left(\mathrm{i} k_{y}\right)^{2}}{2 \kappa^{2} \bar{\Gamma}_{s}} \bar{P}_{n}^{d+}\left(\bar{W}_{n}^{d}+\bar{R}_{n}^{+} \exp \left(-\bar{\Gamma}_{n} d_{n}\right) \bar{W}_{n}^{u}\right),
\end{aligned}
$$

$$
\begin{aligned}
\tilde{G}_{x y ; s}^{e e}= & \frac{\mathrm{i} k_{x} \mathrm{i} k_{y} \Gamma_{n}}{2 \eta_{n} \kappa^{2}} P_{n}^{d-}\left(W_{n}^{d}-R_{n}^{+} \exp \left(-\Gamma_{n} d_{n}\right) W_{n}^{u}\right) \\
& -\frac{\zeta_{s} \mathrm{i} k_{x} \mathrm{i} k_{y}}{2 \kappa^{2} \bar{\Gamma}_{s}} \bar{P}_{n}^{d+}\left(\bar{W}_{n}^{d}+\bar{R}_{n}^{+} \exp \left(-\bar{\Gamma}_{n} d_{n}\right) \bar{W}_{n}^{u}\right), \\
\tilde{G}_{x z ; s}^{e e}= & \frac{\eta_{s} \mathrm{i} k_{x} \Gamma_{n}}{2 \eta_{n} \eta_{s}^{(v)} \Gamma_{s}} P_{n}^{d+}\left(W_{n}^{d}-R_{n}^{+} \exp \left(-\Gamma_{n} d_{n}\right) W_{n}^{u}\right) .
\end{aligned}
$$

\section{REFERENCES}

Abramowitz, M., and I. A. Stegun, 1972, Handbook of mathematical functions, 10th printing ed.: National Bureau of Standards, Applied Mathematics Series.

Abubakar, A., and T. M. Habashy, 2006, A closed-form expression of the electromagnetic tensor Green's functions for a homogeneous TI-anisotropic medium: IEEE Geoscience and Remote Sensing Letters, 3, 447-451, doi: 10.1109/LGRS.2006.874162.

Airy, G. B., 1833, On the phænomena of Newton's rings when formed between two transparent substances of different refractive powers: The London and Edinburgh Philosophical Magazine and Journal of Science, 2, 20-30.
Ali, S. M., and S. F. Mahmoud, 1979, Electromagnetic fields of buried sources in stratified anisotropic media: IEEE Transactions on Antennas and Propagation, 27, 671-678, doi: 10.1109/TAP.1979.1142161.

Gradshteyn, I. S., and I. M. Ryzhik, 1996, Tables of integrals, series, and products, 5th ed.: Academic Press.

Jönsthövel, T. B., C. W. Oosterlee, and W. A. Mulder, 2006, Improving multigrid for 3-D electro-magnetic diffusion on stretched grids: Presented at ECCOMAS European Conference on Computational Fluid Dynamics.

Key, K., 2009, 1D inversion of multicomponent, multifrequency marine CSEM data: Methodology and synthetic studies for resolving thin resistive layers: Geophysics, 74, no. 2, F9-F20, doi: 10.1190/1.3058434.

Kong, F. N., 2007, Hankel transform filters for dipole antenna radiation in a conductive medium: Geophysical Prospecting, 55, 83-89, doi: 10.1111/j .1365-2478.2006.00585.x.

Kong, J., 1972, Electromagnetic fields due to dipole antennas over stratified anisotropic media: Geophysics, 37, 985-996, doi: 10.1190/1.1440321.

Kwon, Y. S., and J. J. H. Wang, 1986, Computation of Hertzian dipole radiation in stratified uniaxial anisotropic media: Radio Science, 21, 891-902, doi: 10.1029/RS021i006p00891.

Lambot, S., E. Slob, and H. Vereecken, 2007, Fast evaluation of zero-offset Green's function for layered media with application to ground-penetrating radar: Geophysical Research Letters, 34, L21405, doi: 10.1029/ 2007 GL031459.

Loseth, L. O., and B. Ursin, 2007, Electromagnetic fields in planarly layered anisotropic media: Geophysical Journal International, 170, 44-80, doi: 10 .1111/j.1365-246X.2007.03390.x.

Michalski, K. A., 2005, Electromagnetic field computation in planar multilayers: in K. Chang, ed.,Encyclopedia of RF and Microwave Engineering, vol. 2, Wiley, 1163-1190.

Mulder, W. A., 2006, A multigrid solver for 3D electromagnetic diffusion: Geophysical Prospecting, 54, 633-649, doi: 10.1111/j.1365-2478.2006 .00558.x.

Mulder, W. A., 2007, A robust solver for CSEM modelling on stretched grids: 69th Annual International Conference and Exhibition, EAGE, Extended Abstracts, D036.

Redheffer, R., 1961, Difference equations and functional equations in transmission-line theory: McGraw-Hill.

Slob, E., J. Hunziker, and W. A. Mulder, 2010, Green's tensors for the diffusive electric field in a VTI half-space: Progress in electromagnetics research: PIER, 107, 1-20, doi: 10.2528/PIER10052807.

Tsang, L., E. Njoku, and J. A. Kong, 1975, Microwave thermal emission from a stratified medium with nonuniform temperature distribution: Journal of Applied Physics, 46, 5127-5133, doi: 10.1063/1.321571.

Ursin, B., 1983, Review of elastic and electromagnetic wave propagation in horizontally layered media: Geophysics, 48, 1063-1081, doi: 10.1190/1 .1441529.

Wait, J., 1951, The magnetic dipole over the horizontally stratified earth: Canadian Journal of Physics, 29, 577-592, doi: 10.1139/p51-060.

Wait, J., 1953, Radiation from a vertical electric dipole over a stratified ground: IEEE Transactions on Antennas and Propagation, AP1, 9-11, doi: 10.1109/T-AP.1953.27320.

Wait, J., 1966, Fields of a horizontal dipole over a stratified anisotropic halfspace: IEEE Transactions on Antennas and Propagation, 14, 790-792, doi: 10.1109/TAP.1966.1138794.

Wang, G. L., C. Torres-Verdín, and S. Gainzero, 2009, Fast simulation of triaxial borehole induction measurements acquired in axially symmetrical and transversely isotropic media: Geophysics, 74, no. 6, E233-E249, doi: 10.1190/1.3261745.

Weiglhofer, W. S., 1990, Dyadic Green's functions for general uniaxial media: IEE Proceedings Part H, Microwaves, Antennas and Propagation, 137, 5-10, doi: 10.1049/ip-h-2.1990.0002.

Xiong, Z., 1989, Electromagnetic fields of electric dipoles embedded in a stratified anisotropic earth: Geophysics, 54, 1643-1646, doi: 10.1190/1 1442633 .

Zhong, L., J. Li, A. Bhardwaj, L. C. Shen, and R. C. Liu, 2008, Computation of triaxial induction logging tools in layered anisotropic dipping formations: IEEE Transactions on Geoscience and Remote Sensing, 46, 11481163, doi: 10.1109/TGRS.2008.915749. 